${ }^{1}$ UPR 9034 Evolution, génomes et spéciation, CNRS, Gif sur Yvette cedex, France; ${ }^{2}$ INRA, Centre de Biologie et de Gestion des Populations, Montferrier-sur-Lez, France; ${ }^{3}$ Institut Curie, Orsay, France; ${ }^{4}$ IRD, UR R072, c/o CNRS UPR 9034, Gif sur Yvette cedex, France

\title{
A phylogeny of Drosophilidae using the Amyrel gene: questioning the Drosophila melanogaster species group boundaries
}

\author{
J.-L. Da Lage ${ }^{1}$, G. J. Kergoat ${ }^{2}$, F. Maczkowiak ${ }^{3}$, J.-F. Silvain ${ }^{4}$, M.-L. Cariou ${ }^{1}$ and D. Lachaise ${ }^{1 *}$
}

\begin{abstract}
In this study, the phylogenetic relationships of 164 species of the family Drosophilidae are discussed, using the Amyrel gene, a member of the $\alpha$-amylase multigene family. This study focuses on numerous species groups in the subgenera Sophophora and Drosophila of the genus Drosophila but also includes other closely related genera. Nucleotide data were analysed by several methods: maximum parsimony, neighbour joining, maximum likelihood and Bayesian inference. Heterogeneity of base composition (mainly low GC contents in the species groups willistoni and saltans) has been addressed. In all analyses, the genus Drosophila appeared paraphyletic. The subgenus Sophophora clearly appeared to be a monophyletic group, showing well-resolved clades, with the Neotropical groups arising in a basal position. Here, it is proposed to raise the species subgroups ananassae and montium to the rank of species group, and to restrict the melanogaster species group to the melanogaster subgroup plus the 'Oriental' subgroups, among which the suzukii subgroup is polyphyletic. Some related genera such as Zaprionus, Liodrosophila, Scaptomyza and Hirtodrosophila are clustered with, or inside the subgenus Drosophila, which is therefore paraphyletic and should be reviewed.
\end{abstract}

Key words: Molecular phylogeny - Zaprionus - ananassae species group - montium species group - base composition bias - Sophophora

\section{Introduction}

In a few years, 100 years of using Drosophila melanogaster as a laboratory model will be celebrated. This little fly has been widely used in all fields of biology, including genetics, cell biology, ecology, physiology, developmental biology and evolution. Among other obvious, convenient and scientific features of D. melanogaster, which make it suitable for this purpose, it has the advantage of belonging to a genus comprising more than 3500 species distributed worldwide in a variety of climates and ecological niches (Powell 1997; Ashburner et al. 2005). This has made it a gold mine for research into evolutionary genetics and ecology. The occurrence of a number of pairs or complexes of sibling species among the genus Drosophila has led some researchers to focus on speciation and its mechanisms (Powell 1997, p. 213-266; Wu 2001; Coyne and Orr 2004).

More recently, in developmental genetics for instance, it has become necessary to compare between more and less closely related species (DeSalle and Grimaldi 1993; Gompell and Carroll 2003). Thus, apart from the interest of phylogenetic relationships per se between drosophilid species, it has become even more crucial to have accurate and extensive phylogenetic trees, covering as many species as possible.

Following the pioneer works of Throckmorton (1975) or Grimaldi (1990), several attempts to reconstruct species trees of Drosophila have been published since the early 1990s. Molecular data have been used to produce numerous trees, using rDNA (Pélandakis et al. 1991; Pélandakis and Solignac 1993), mtDNA (DeSalle 1992; Gleason and Powell 1997; Goto and Kimura 2001; Kastanis et al. 2003), nuclear genes or combined data (e.g. Powell 1997; Durando et al. 2000; Kopp and True 2002; O'Grady and Kidwell 2002; Remsen and O'Grady 2002; Robe et al. 2005), sometimes including morphology (Remsen and O'Grady 2002; Schawaroch 2002).

*Dr Daniel Lachaise died on 2 July 2006, suddenly and unexpectedly. He has contributed very much to this article.
These works have usually focused on particular taxonomic groups, or inversely, at a larger scale, on relationships between Drosophila subgenera and closely related genera. Molecular studies have often been hindered by the varied evolutionary patterns displayed by the genes used, such as composition bias and lineage-specific substitution rates (Tarrio et al. 2001).

Some consistent data are now available, such as the monophyly of the subgenus Sophophora (O'Grady and Kidwell 2002, but see Powell 1997; Katoh et al. 2000), relationships between some species groups and the existence of deep (ancient) radiations within the subgenus Drosophila. There is still no large-scale phylogeny of Drosophilidae, including distant species, but also showing more details in some species groups. An attempt was made to produce such a phylogeny using sequence data from the Amyrel gene.

Amyrel is a nuclear gene, a paralogue of the $\alpha$-amylase (Amy) genes. The usefulness of Amy genes in molecular phylogenetics is usually limited by the fact that they are mostly found in multiple copies, unless each copy has been identified in each species (see Inomata et al. 1997; Zhang et al. 2003; also Kopp 2006). In contrast, Amyrel is a single-copy gene (Da Lage et al. 1998). It has $40 \%$ divergence with Amy in amino acids and is easily distinguished from Amy. It was first identified in species of the subgenus Sophophora, and was originally thought to be restricted to this subgenus (Da Lage et al. 1998). Further investigations have shown that Amyrel is also present in other families of the Muscomorpha clade (Da Lage et al. 2002; Maczkowiak and Da Lage, 2006). In Drosophila, the coding sequence is about $1470-1485$ bp in length, with a single short intron located near the middle of the gene (position 655 in D. melanogaster). Amyrel is an attractive candidate for use in producing molecular phylogenies because it can be entirely amplified by PCR using external primers. Conserved regions exist in $5^{\prime}$ and $3^{\prime}$, which make it possible to design suitable primers (Da Lage et al. 1998). Amyrel has been already used to resolve phylogenetic relationships (Cariou 
et al. 2001; Kopp 2006). It is also informative at the intraspecific level in several species (M.L. Cariou, unpublished data).

A single-gene phylogeny with a large amount of original data is presented here. This single-gene approach has been chosen to be retained in order to increase the number of species covered by this study, rather than a multicharacter strategy, with a lesser number of taxa. Recent works on various organisms (Gontcharov et al. 2004; May-Collado and Agnarsson 2006) have suggested that dense taxon sampling on a single gene is relevant for improving phylogenetic accuracy. In addition, recently, Kopp (2006) used Amyrel in a multigene approach of the D. melanogaster species group. Clearly, his Amyrel tree was the most congruent with the retained consensus tree. Despite the drawbacks of using only one gene, this work has the advantage of covering a large number of species, some of which were never included in previously published molecular phylogenies. The complete sequences for 146 species have been obtained. Partial sequences have also been obtained from 18 other species.

\section{Materials and Methods \\ Flies and DNA extraction}

Live specimens of Drosophilidae species were available from the Gif laboratory collection, which also contained frozen and alcoholpreserved specimens of other species. The species are listed in Table 1. Genomic DNAs from single individuals were prepared as described previously (Gloor and Engels 1992).

\section{Amyrel amplification}

External primers were designed for the subgenus Sophophora, using the conserved regions described in Da Lage et al. (1998): RELZONE2 (forward) TCGTAAATTGGACCCAAGCG; RELAVALREV (reverse) CATACATTATGTGCGTTCG. Using this pair of primers, it has been found that reducing the elongation temperature yielded more products, perhaps because of the low $T_{\mathrm{m}}$ of the reverse primer. The cycles were as follows: denaturation $94^{\circ} \mathrm{C} 30 \mathrm{~s}$; annealing $53^{\circ} \mathrm{C} 1 \mathrm{~min}$; extension $65^{\circ} \mathrm{C} 2 \mathrm{~min} ; 40$ cycles. The size of amplified DNA was ca. $1.7 \mathrm{~kb}$. For the non-Sophophora species, no data were available pertaining to the conservation of external regions. Amplifications with internal primers previously used in sequencing were obtained thus: 1U (forward) GTTCACCTCTTCGAGTGG, REV1230 (reverse) TTGCTGCCRTTRTCCCACC. The Amyrel gene of Drosophila virilis was then cloned from a genomic minilibrary; and the genes of Drosophila funebris and Hirtodrosophila confusa were completed using the Universal Genome Walker Kit (Clontech, Mountain View, CA). An alignment of these three sequences enabled to design external primers that could be used for the non-Sophophora species: ZONE2BIS (forward) GTAAATNGGNNCCACGCGAAG; RELAVBIS (reverse) GCATTTGTACCGTTT GTGTCGTTATCG. However, in many cases, it was more convenient to obtain two shorter overlapping fragments, ca. $1 \mathrm{~kb}$ each, between ZONE2BIS and RELREV + (GTTCCCCAGCTCTGCAGCC) for the left part and between RELUDIR (TGGATGCNGCCAAGCACATGGC) and RELAVBIS for the right part. The positioning of the primers used in this study is shown in Fig. 1.

When possible, PCR products were directly sequenced on an ABI373 sequencer, otherwise they were cloned in the pGEM-T plasmid vector (Promega, Madison, WI). In this case, several clones were sequenced and one has been retained in the data set.

The Amyrel gene from the medfly Ceratitis capitata (Tephritidae) was cloned from a minilibrary using a fragment of the $A m y$ gene of this species as a probe. All the sequences were deposited in GenBank. The accession numbers are shown in Table 1 .

\section{Sequence alignment}

The DNA sequences, excluding introns, were translated, and then the protein sequences were aligned using CLUSTALW (Thompson et al. 1994).
This alignment served as a guide to manually align the DNA sequences. Ambiguities putatively occurred only in the signal peptide, about 20 amino acids in length, which is highly variable between distant species. A total of 164 taxa were aligned (1515 positions). Using a chi-square test implemented in PAUP* version 4.0b10 (Swofford 2002), significant base composition heterogeneity was detected between taxa.

\section{Phylogenetic reconstruction methods}

Several methods were used to reconstruct the phylogenetic relationships of the species sampled. PAUP* version $4.0 \mathrm{~b} 10$ (Swofford 2002) was used to carry out both neighbour joining (NJ) and maximum parsimony (MP) analyses. Bayesian inference (BI) analyses were conducted using MrBayes version 3.1.1 (Huelsenbeck and Ronquist 2001), whereas PHYML version 2.1b (Guindon and Gascuel 2003) was used for maximum likelihood (ML) inference. All these analyses used two outgroups: the close relative Leucophenga maculata (Drosophilidae, Steganinae) (accession number DQ021938) and the more distant C. capitata (Tephritidae) (accession number AF146758).

Neighbour joining analyses were conducted as preliminary analyses with both Kimura two-parameters (K2P) and LogDet (LD) corrections. The latter correction was used in an attempt to better take into account the significant heterogeneity in base composition between taxa (Galtier and Gouy 1998).

Given the large number of taxa in the data set, all parsimony analyses were performed using heuristic search option (tree bisection and reconnection (TBR), random sequence addition). To better explore the tree space when working with a large number of taxa, 1000 random addition replicates were used and TBR branch swapping was limited to a maximum of 500 trees (i.e. MaxTrees set to 500), as proposed by Sorenson (1999). Both unweighted and weighted analyses were conducted. Weighted analyses were carried out, in which the third codon positions were underweighted, to minimize the effect of transitions that may accumulate at high frequency at the third codon position because of the degeneracy of the genetic code. To determine the sensitivity of the phylogenetic reconstructions to weighting, the weighting of the first and second codon positions has been progressively increased over the third codon positions using three differential weightings of the three codon positions $(2: 2: 1,4: 4: 1$ and $10: 10: 1)$. Given the fact that transitions are more frequent than transversions (Brown et al. 1982), a weighted analysis of the data set was also performed following Hillis et al. (1994), who recommended increasing the resolution of parsimony analyses by downweighting transitions. Since similar results are generally obtained when using different numbers to downweight transitions versus transversions (Barker and Lanyon 2000), a single weighting scheme was used for downweighting transitions (transversions were weighted twice over transitions). The robustness of topologies was assessed by bootstrap procedures (1000 replicates) and by estimating Bremer support (BS) values (Bremer 1994) using TreeRot version 2.0 (Sorenson 1999).

Before carrying out the BI and ML analyses, Modeltest version 3.06 (Posada and Crandall 1998) was used to determine the best-fit substitution model for the data under a likelihood framework. The Akaike information criterion selected the general time reversible (GTR) model including the proportion of invariable sites and the gamma distribution of rate variation among sites (GTR $+\mathrm{I}+\Gamma$ : Lanave et al. 1984; Yang 1994; $\mathrm{Gu}$ et al. 1995) as the best-fit evolutionary model. For BI analyses, four distinct 2000000 generation runs were conducted using the Markov Chain Monte Carlo (MCMC) algorithm implemented in MrBayes (four incrementally heated chains were used with a GTR $+\mathrm{I}+\Gamma$ model and trees were saved to a file every 100 generations). Identical topologies were recovered from all four runs. A burn-in period of 100000 generations was identified graphically by plotting likelihood values for each generation. The results were presented in the form of a $50 \%$ majorityrule consensus tree (in which trees corresponding to the burn-in period were discarded) and the support for the nodes of the tree were given by posterior probability estimates of clades. The ML analysis was performed using an input tree generated by an NJ heuristic search (BIONJ option in PHYML), the GTR $+\mathrm{I}+\Gamma$ model and all parameters estimated from the data. Branch support was assessed by 1000 bootstrap replicates using ML distances as implemented in PAUP*. 
Table 1. List of Drosophila taxa used in this study. Asterisks indicate partial Amyrel sequences. The new terminology proposed in this article was used in the former melanogaster species group. Group and subgroup taxonomy follows that proposed by Bächli (1999)

\begin{tabular}{|c|c|c|c|c|c|}
\hline Subgenus (or genus) & Group & Subgroup & Species & Origin & Accession number \\
\hline \multirow[t]{67}{*}{ Drosophila } & annulimana & & aracataca (Vilela and Val, 1983) & Costa Rica & AY733052 \\
\hline & & & ararama (Pavan and Cunha, 1947) & & AY733048 \\
\hline & & & gibberosa (Patterson and Mainland, 1943) & Mexico & AY733041 \\
\hline & & & talamancana (Wheeler, 1968) & El Salvador & AY736509 \\
\hline & bromeliae & & bromeliae (Sturtevant, 1921) & Guadeloupe & AY733049 \\
\hline & cardini & cardini & cardini (Sturtevant, 1916) & Martinique & AF462599 \\
\hline & & cardini & neomorpha (Heed and Wheeler, 1957) & & AY736481 \\
\hline & & cardini & polymorpha (Dobzhansky and Pavan, 1943) & Florida & AY736495 \\
\hline & & dunni & arawakana $($ Heed, 1962) & Guadeloupe & AF491630 \\
\hline & & dunni & caribiana (Heed, 1962) & Martinique & AY733050 \\
\hline & dreyfusi & & camargoi (Pavan, 1950) & British Guyana & AF462598 \\
\hline & funebris & funebris & funebris (Fabricius, 1787) & France & AF335557 \\
\hline & guarani & guarani & guaru (Dobzhansky and Pavan, 1943) & & AF491631 \\
\hline & histrio & & sternopleuralis (Okada and Kurokawa, 1957) & Japan & AY736505 \\
\hline & immigrans & hypocausta & hypocausta (Osten Sacken, 1882) & & AY733043 \\
\hline & & hypocausta & rubida (Mather,1960) & New Ireland & AY736502 \\
\hline & & hypocausta & siamana (Ikeda et al. 1983) & Thailand & AY736504 \\
\hline & & immigrans & formosana (Duda, 1926) & & AF462601 \\
\hline & & immigrans & immigrans (Sturtevant, 1921) & New Caledonia & AF491632 \\
\hline & & immigrans & ruberrima (de Meijere, 1911) & & AY736501 \\
\hline & & immigrans & ustulata (de Meijere, 1908) & Indonesia & AY736541* \\
\hline & & nasuta & albomicans (Duda, 1923) & Thailand & AF462595 \\
\hline & & nasuta & kepulauana (Wheeler, 1969) & & AY733044 \\
\hline & & nasuta & nasuta (Lamb, 1914) & & AY733059 \\
\hline & & nasuta & pallidifrons (Wheeler, 1969) & New Caledonia & AY736486 \\
\hline & & nasuta & sulfurigaster (Duda, 1923) & New Caledonia & AY736508 \\
\hline & & & trilimbata (Bezzi, 1928) & & AY736512 \\
\hline & melanica & & melanica (Sturtevant, 1916) & Arizona & AY733056 \\
\hline & & & tsigana (Burla and Gloor, 1952) & & AY736513 \\
\hline & mesophragmatica & & pavani (Brncic, 1957) & Argentina & AY736490 \\
\hline & Modified mouthparts & mimica & mimica (Hardy, 1965) & Hawaii & AY736537* \\
\hline & pallidipennis & & pallidipennis (Patterson and Mainland, 1943) & Panama & AY736487 \\
\hline & Picture wing & grimshawi & grimshawi (Oldenberg, 1914) & Hawaii & AY736533* \\
\hline & polychaeta & & daruma (Okada, 1956) & Okinawa & AY736532* \\
\hline & & & iri (hirtipes) (Burla, 1954) & Congo & AF491633 \\
\hline & & & latifshahi (Gupta and Ray-Chaudhury, 1970) & India & AY736536* \\
\hline & & & polychaeta (Patterson and Wheeler, 1942) & Guadeloupe & AY736494 \\
\hline & quinaria & & angularis (Okada, 1956) & Japan & AY736530* \\
\hline & & & kuntzei (Duda, 1924) & & AF491634 \\
\hline & & & limbata (von Roser, 1840) & France & AF491636 \\
\hline & & & nigromaculata (Kikkawa and Peng, 1938) & Japan & AY736483 \\
\hline & & & palustris (Spencer, 1942) & & AY736538* \\
\hline & & & phalerata (Meigen, 1830) & & AY736492 \\
\hline & & & subpalustris (Spencer, 1942) & & AY736539* \\
\hline & & & transversa (Fallen, 1823) & & AY736511 \\
\hline & repleta & hydei & hydei (Sturtevant, 1921) & & AY733042 \\
\hline & & hydei & hydeoides (Patterson and Wheeler, 1942) & & AY736534* \\
\hline & & mulleri & nigrodumosa (Wasserman and Fontdevila, 1990) & Venezuela & AY736482 \\
\hline & & mulleri & wheeleri (Patterson and Alexander, 1952) & & AY736514 \\
\hline & & repleta & limensis (Pavan and Patterson, 1947) & Peru & AY736479 \\
\hline & & repleta & repleta (Wollaston, 1858) & France & AY736496 \\
\hline & testacea & & testacea (von Roser, 1840) & Caucase & AY736510 \\
\hline & tripunctata & iii & mediopictoides (Heed and Wheeler, 1957) & Panama & AY733055 \\
\hline & & iv & albirostris (Sturtevant, 1921) & & AY733047 \\
\hline & & iv & metzii (Sturtevant, 1921) & & AY744447 \\
\hline & tumiditarsus & & repletoides $(\mathrm{Hsu}, 1943)$ & China & AY736500 \\
\hline & virilis & americana & americana (Spencer, 1938) & & AY736529* \\
\hline & & americana & americana texana (Patterson, 1940) & & AY736540* \\
\hline & & virilis & virilis (Sturtevant, 1916) & Spain & AF136603 \\
\hline & & & kanekoi (Watabe and Higuchi, 1979) & Japan & AY736535* \\
\hline & & & littoralis (Meigen, 1830) & & AY733045 \\
\hline & & & lummei (Hackman, 1972) & Finland & AY733046 \\
\hline & & & novamexicana (Patterson, 1941) & & AY736484 \\
\hline & Ungrouped & & adamsi (Wheeler, 1959) & & AY736528* \\
\hline & Ungrouped & & aracea (Heed and Wheeler, 1957) & Mexico & AY736531* \\
\hline & Ungrouped & & pruinosa (Duda, 1940) & Madagascar & AY756177 \\
\hline & ananassae & & ananassae (Doleschall, 1858) & Ivory Coast & AF024691 \\
\hline
\end{tabular}


Table 1. (Continued)

\begin{tabular}{|c|c|c|c|c|c|}
\hline Subgenus (or genus) & Group & Subgroup & Species & Origin & Accession number \\
\hline & \multirow{67}{*}{ melanogaster } & \multirow{67}{*}{$\begin{array}{l}\text { elegans } \\
\text { elegans } \\
\text { eugracilis } \\
\text { ficusphila } \\
\text { ficusphila } \\
\text { flavohirta } \\
\text { melanogaster } \\
\text { melanogaster } \\
\text { melanogaster } \\
\text { melanogaster } \\
\text { melanogaster } \\
\text { melanogaster } \\
\text { melanogaster } \\
\text { melanogaster } \\
\text { melanogaster } \\
\text { suzukii } \\
\text { suzukii } \\
\text { suzukii } \\
\text { takahashii } \\
\text { takahashii } \\
\text { takahashii }\end{array}$} & atripex (Bock and Wheeler, 1972) & Thailand & U96154 \\
\hline & & & bipectinata (Duda, 1923) & Thailand & AF136936 \\
\hline & & & ercepeae (Tsacas and David, 1975) & Réunion & U96155 \\
\hline & & & lachaisei (Tsacas, 1984) & Sao Tomé & AY736478 \\
\hline & & & malerkotliana pallens (Bock and Wheeler, 1972) & Borneo & AY733054 \\
\hline & & & malerkotliana (Parshad and Paika, 1964) & Madagascar & AY733053 \\
\hline & & & merina (Tsacas, 1997) & Madagascar & AY733057 \\
\hline & & & monieri (McEvey et al. 1987) & Moorea & AF250052 \\
\hline & & & ochrogaster (Chassagnard, 1992) & New Caledonia & AY736485 \\
\hline & & & pallidosa (Bock and Wheeler, 1972) & Samoa & AF136931 \\
\hline & & & papuensis-like & New Guinea & AY736488 \\
\hline & & & parabipectinata $($ Bock, 1971) & Mauritius & AY736489 \\
\hline & & & phaeopleura (Bock and Wheeler, 1972) & Fiji & AY736491 \\
\hline & & & pseudoananassae pseudoananassae (Bock, 1971) & Thailand & AY736498 \\
\hline & & & pseudoananassae nigrens (Bock and Wheeler, 1972) & Thailand & AY736497 \\
\hline & & & vallismaia (Tsacas, 1984) & Seychelles & AY744446 \\
\hline & & & varians (Bock and Wheeler, 1972) & Philippines & AF136937 \\
\hline & & & elegans (Bock and Wheeler, 1972) & Philippines & AF 136930 \\
\hline & & & subelegans (Okada, 1988) & & AY736507 \\
\hline & & & eugracilis (Bock and Wheeler, 1972) & Thailand & AF250055 \\
\hline & & & ficusphila (Kikkawa and Peng, 1938) & Taiwan & AF462600 \\
\hline & & & levii (Tsacas, 1988) & New Caledonia & AF491635 \\
\hline & & & flavohirta (Malloch, 1924) & Australia & AY733051 \\
\hline & & & erecta (Tsacas and Lachaise, 1974) & Ivory Coast & AF039562 \\
\hline & & & mauritiana (Tsacas and David, 1974) & Mauritius & U96157 \\
\hline & & & melanogaster (Meigen, 1830) & Canton $\mathrm{S}$ & AF022713 \\
\hline & & & orena (Tsacas and David, 1978) & Cameroon & U96158 \\
\hline & & & santomea (Lachaise and Harry, 2000) & Sao Tomé & AY736503 \\
\hline & & & sechellia (Tsacas and Bächli, 1981) & Seychelles & AF039558 \\
\hline & & & simulans (Sturtevant, 1919) & Réunion & U96159 \\
\hline & & & teissieri (Tsacas, 1971) & Congo & AF039557 \\
\hline & & & yakuba (Burla, 1954) & Cameroon & AF039561 \\
\hline & & & biarmipes (Malloch, 1924) & India & AF462597 \\
\hline & & & lucipennis (Lin, 1972) & Taiwan & AF251138 \\
\hline & & & mimetica (Bock and Wheeler, 1972) & Malaysia & AY733058 \\
\hline & & & lutescens (Okada, 1975) & Japan & AF491637 \\
\hline & & & pseudotakahashii (Mather, 1957) & Australia & AY736499 \\
\hline & & & takahashii (Sturtevant, 1927) & Thailand & U96161 \\
\hline & & & asahinai (Okada, 1964) & Japan & AF250051 \\
\hline & & & auraria (Peng, 1937) & China & U96163 \\
\hline & & & bakoue (Tsacas and Lachaise, 1974) & Bénin & U96162 \\
\hline & & & barbarae (Bock and Wheeler, 1972) & Thailand & AF250053 \\
\hline & & & biauraria (Bock and Wheeler, 1972) & Japan & AF 136932 \\
\hline & & & bicornuta (Bock and Wheeler, 1972) & Taiwan & AF 136933 \\
\hline & & & bocqueti (Tsacas and Lachaise, 1974) & Ivory Coast & AF049092 \\
\hline & & & burlai (Tsacas and Lachaise, 1974) & Ivory Coast & AF250059 \\
\hline & & & cauverii (Muniyappa et al. 1982) & India & AF251137 \\
\hline & & & chauvacae (Tsacas, 1984) & Madagascar & AF250056 \\
\hline & & & davidi (Tsacas, 1975) & Congo & AF251139 \\
\hline & & & diplacantha (Tsacas and David, 1977) & Cameroon & AF251142 \\
\hline & & & dossoui (Chassagnard, 1991) & Bénin & U96164 \\
\hline & & & greeni (Bock and Wheeler, 1972) & Ivory Coast & AF462602 \\
\hline & & & jambulina (Parshad and Paika, 1964) & India & AF 174489 \\
\hline & & & kikkawai (Burla, 1954) & Madagascar & U96156 \\
\hline & & & leontia (Tsacas and David, 1977) & India & AF250058 \\
\hline & & & lini (Bock and Wheeler, 1972) & Taiwan & AF039559 \\
\hline & & & malagassya (Tsacas and Rafael, 1982) & Madagascar & AF250057 \\
\hline & & & nagarholensis (Prakash and Reddy, 1980) & India & AF250054 \\
\hline & & & nikananu (Burla, 1954) & Cameroon & AF251136 \\
\hline & & & nsp. montium (Lachaise, unpublished data) & Príncipe Island & \\
\hline & & & punjabiensis (Parshad and Paika, 1964) & Thailand & U96165 \\
\hline & & & quadraria (Bock and Wheeler, 1972) & Taiwan & AF 136934 \\
\hline & & & rufa (Kikkawa and Peng, 1938) & Japan & AF 136935 \\
\hline & & & serrata (Malloch, 1927) & Australia & AF069756 \\
\hline & & & triauraria (Bock and Wheeler, 1972) & Japan & AF251141 \\
\hline & & & tsacasi (Bock and Wheeler, 1972) & Ivory Coast & AF251134 \\
\hline & & & bocqueti-like (Lachaise, unpublished data) & Congo & AF251131 \\
\hline
\end{tabular}


Table 1. (Continued)

\begin{tabular}{|c|c|c|c|c|c|}
\hline Subgenus (or genus) & Group & Subgroup & Species & Origin & Accession number \\
\hline & & & vulcana (Graber, 1957) & Zimbabwe & AF251132 \\
\hline & obscura & obscura & bifasciata (Pomini, 1940) & Finland & AF251135 \\
\hline & & obscura & imaii (Moriwaki and Okada, 1967) & Japan & AF251133 \\
\hline & & obscura & subobscura (Collin, 1936) & France & U79724 \\
\hline & & pseudoobscura & pseudoobscura (Frolova, 1929) & Arizona & U82556 \\
\hline & & microlabis & kitumensis (Tsacas, 1985) & Kenya & AF306718 \\
\hline & & affinis & affinis (Sturtevant, 1916) & Georgia (USA) & AF037353 \\
\hline & saltans & & neocordata (Magalhaes, 1956) & Brazil & AY736480 \\
\hline & & & sturtevanti (Duda, 1927) & Guadeloupe & AY736506 \\
\hline & willistoni & & nebulosa (Sturtevant, 1916) & Guadeloupe & AY733060 \\
\hline & & & tropicalis (Burla and Cunha, 1949) & El Salvador & AF251140 \\
\hline & & & willistoni (Sturtevant, 1916) & Guadeloupe & AF039560 \\
\hline Chymomyza & & & amoena (Loew, 1862) & Michigan & AY736544* \\
\hline Hirtodrosophila & & & confusa (Staeger, 1844) & France & AF335559 \\
\hline Hirtodrosophila & & & pictiventris (Duda, 1925) & & AY736493 \\
\hline Liodrosophila & & & aerea (Okada, 1956) & Japan & AY736477 \\
\hline Mycodrosophila & & & sp. & Russia (Sotchi) & AY736543* \\
\hline Scaptodrosophila & & & finitima (Lamb, 1914) & Madagascar & AY736527 \\
\hline Scaptodrosophila & & & bryani (Malloch, 1934) & Moorea & AY756178* \\
\hline Scaptomyza & & & pallida (Zettzerstedt, 1847) & France & AY736542 \\
\hline Anaprionus & & & bogoriensis (Mainx, 1958) & Thailand & AY736516 \\
\hline Anaprionus & & & lineosus (Walker, 1860) & India & AY736521 \\
\hline Zaprionus & & & badyi (Burla, 1954) & Ivory Coast & AY736515 \\
\hline Zaprionus & & & cercus (Chassagnard and McEvey, 1992) & Madagascar & AY736517 \\
\hline Zaprionus & & & ghesquierei (Collart, 1937) & Cameroon & AY736518 \\
\hline Zaprionus & & & inermis (Collart, 1937) & Cameroon & AY736519 \\
\hline Zaprionus & & & kolodkinae (Chassagnard and Tsacas, 1987) & Madagascar & AY736520 \\
\hline Zaprionus & & & mascariensis (Tsacas and David, 1975) & Madagascar & AY736522 \\
\hline Zaprionus & & & sepsoides (Duda, 1939) & Central Africa & AY736523 \\
\hline Zaprionus & & & sg vittiger & Madagascar & AY736526 \\
\hline Zaprionus & & & tuberculatus (Malloch, 1932) & Ivory Coast & AY736524 \\
\hline Zaprionus & & & verruca (Chassagnard and McEvey, 1992) & Madagascar & AY736525 \\
\hline
\end{tabular}

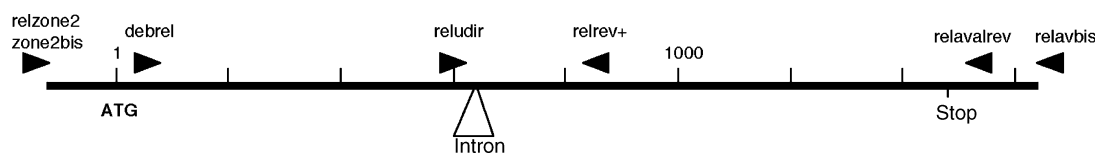

Fig. 1. Diagram of the Amyrel gene showing the positions of the primers used for amplification and sequencing; the intron position is also shown

\section{Results}

\section{The data set}

Complete sequences were obtained for 146 species and partial sequences for 18 species. All drosophilid species have an intron at the same position. This intron is almost always short, around $60 \mathrm{bp}$ (minimum size $46 \mathrm{bp}$, maximum $284 \mathrm{bp}$, median $57 \mathrm{bp}$, mean $60 \mathrm{bp}$ ). The Amyrel sequence of $C$. capitata has two introns: the first one is located at the same position as the intron of classical Amy genes of Drosophila (Da Lage et al. 1996) and the second intron is homologous to the single intron of Amyrel genes of Drosophila. In C. capitata, the reading frame is much longer than that in Drosophila species. It has been truncated in the study. Many species were found to be polymorphic for Amyrel, that is, two haplotypes were found after cloning. High variability was found in both the length and the sequence of the signal peptide. An additional codon, in the last third of the sequence, was shared by two species of the obscura group, Drosophila imaii and Drosophila bifasciata.

The base composition of Amyrel is generally GC rich with a mean of $56 \%$. The GC content at the third position of codons
(GC3) is more variable between species groups, with a mean of $72 \%$, but with extremes as low as $38.7 \%$ (Drosophila sturtevanti) and as high as $87.3 \%$ (Drosophila lucipennis) (Fig. 2). Indeed, the Neotropical groups of the subgenus Sophophora, i.e. the willistoni and saltans species groups, are strikingly GC poor, as has already been reported for several other genes (Rodriguez-Trelles et al. 2000). It is important to keep this in mind when looking at the phylogenetic trees below.

\section{General topology}

The trees obtained by the different tree-building methods have been rooted with L. maculata and C. capitata. Two topologies, produced by MP 4: 4:1 and BI, respectively, have been kept for illustration; but for better clarity only the BS values for MP 4:4:1 have been chosen for discussion (note that these values can be very high as they reflect the $4: 4: 1$ weighted matrix). The results of the other weighting schemes or other reconstruction methods used will be discussed in the text. Two main clades emerged from both methods (Fig. 3). Clade 1 


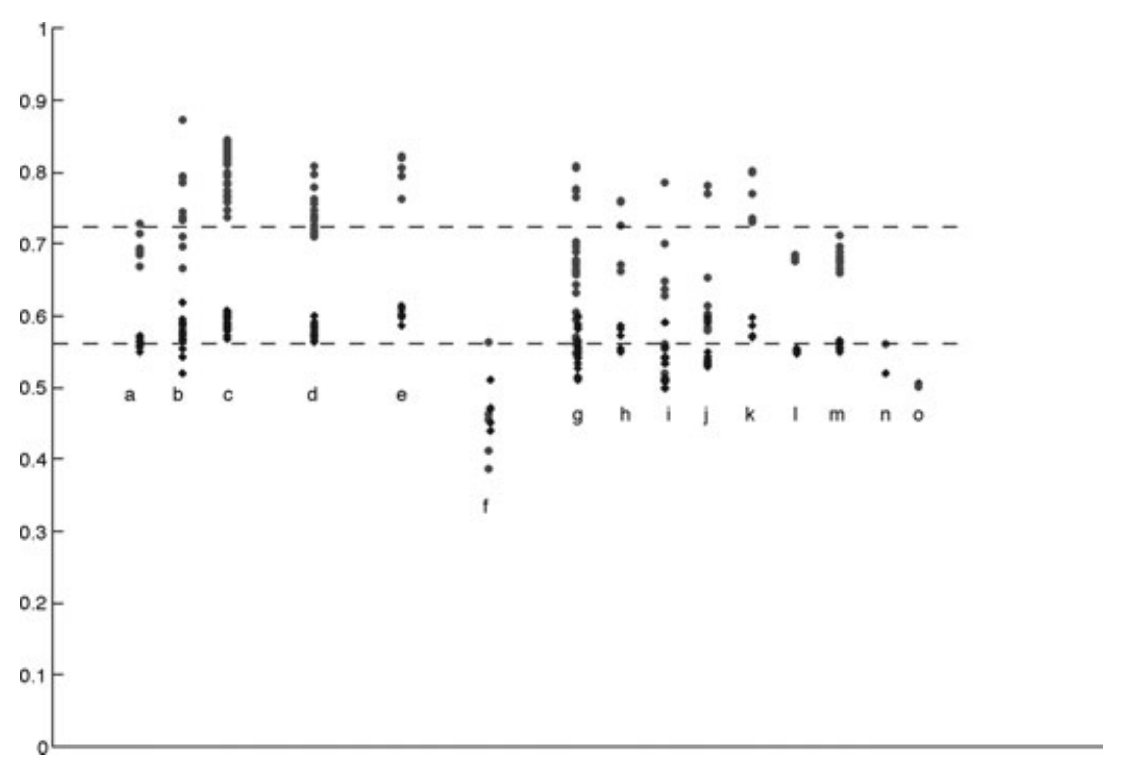

Fig. 2. Base composition of the Amyrel genes (complete coding sequences) of the species studied, grouped by taxonomic affinities. Lozenges are global GC contents; circles are GC content at the third codon positions. Dashed lines show the means for each parameter. a: melanogaster subgroup, b: Oriental subgroups, c: montium subgroup (new species group), d: ananassae subgroup (new species group), e: obscura group, f: Neotropical groups, g: various species in the subgenus Drosophila, h: quinaria group, i: immigrans group, $\mathrm{j}$ : Zaprionus, k: repleta group, 1 : annulimana group, m: virilis group, n: S. finitima, o: C. capitata

(a)

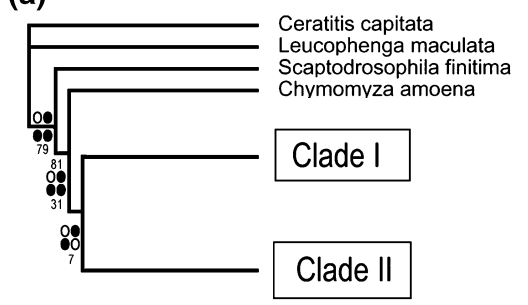

(b)

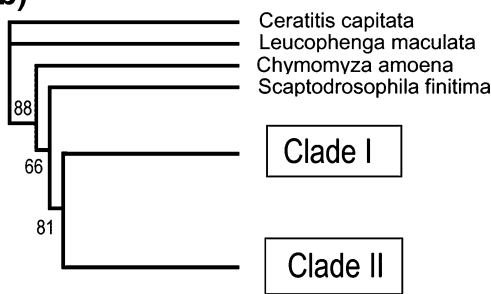

Fig. 3. Basal phylogenetic relationships obtained through parsimony (a) and Bayesian inference (b) analyses. Both inference methods recovered two identical large clades (labelled clade 1 and clade 2), the phylogenetic relationships of which are shown in detail in the corresponding figures (see Figs 4 and 5 for parsimony analyses and Figs 6 and 7 for Bayesian inference analysis). Legends are as in Figs 4 and 6

corresponds to the subgenus Sophophora, whereas clade 2 corresponds to the subgenus Drosophila and several taxa considered as distinct genera. As a consequence, the genus Drosophila clearly appears to be paraphyletic. More detailed results of MP analyses are shown in Figs 4 and 5. A tree obtained with $4: 4: 1$ weighting is drawn, with an indication of its congruence with alternative MP analyses The unweighted MP (MP uw) analysis shows a paraphyletic subgenus Sophophora, with the Neotropical species groups willistoni and saltans branching off at the base of the tree. More over, the tree shows that Sophophora is included in a nested succession of clades belonging to the subgenus Drosophila. For instance, the polychaeta group is the sister group of a clade composed of the members of species groups melanogaster + obscura, which in turn is a sister of the virilis-repleta clade. Most of the deep nodes are poorly supported, except a highly supported

(C) 2007 The Authors J Zool Syst Evol Res (2007) 45(1), 47-63 Journal compilation @ 2007 Blackwell Verlag, Berlin melanogaster + obscura node (bootstrap value of $99 \%$ ). This general topology is clearly incongruent with phylogenetic relationships already known to exist, especially for deeper nodes. MP $4: 4: 1$ (Figs 3a, 4 and 5) and the two other codon-position-based weighted MP analyses (i.e. $2: 2: 1$ and $10: 10: 1)$ recover two main clades: (i) the subgenus Sophophora, including the Neotropical groups, with a bootstrap value of $56 \%$ and $\mathrm{BS}$ of 18 (clade 1) and (ii) the subgenus Drosophila + the genera Zaprionus, Hirtodrosophila, Liodrosophila, Scaptomyza, Mycodrosophila, clustered together with a bootstrap support of $96 \%$ and BS of 87 (clade 2). In a more basal position, Chymomyza amoena and Scaptodrosophila finitima were recovered in a similar way to the MP uw analysis. The BI tree (Figs 3b, 6 and 7) also shows two main clades, with Scaptodrosophila appearing more closely related to clades 1 and 2 compared with Chymomyza. Neighbour joining analyses yield essentially the same general topology as weighted MP, except that the Neotropical species groups are located at the base of the tree, with Ch. amoena and S. finitima clustered together (bootstrap value of $65 \%$ ). The results of K2P and LD analyses only differ by the placement of Drosophila nebulosa (see below) and by their somewhat distinct bootstrap support values. The ML analysis yields a general topology, which is mostly congruent with the result of the MP uw analysis. The two latter topologies only differ by the alternative placement of the Neotropical species groups, which are clustered with the subgenus Sophophora and related to S. finitima and Ch. amoena in the ML analysis. Thus, it can be summarized that a number of methods place the Neotropical groups willistoni and saltans just above the root of the tree (see below). The clade 2, composed by members of the subgenus Drosophila and some taxa hitherto classified as distinct genera, is recovered in most analyses, with a high support for both MP $4: 4: 1$ and BI analyses. Interestingly, in MP reconstructions, the same phylogenetic hypothesis was found using all three distinct weighting schemes, in which the third position of codon was downweighted. This surprising result is consistent with previous observations made by Barker and Lanyon (2000), and suggests, for the data set pertaining to this study at least, that the exact value used for downweighting third codon 

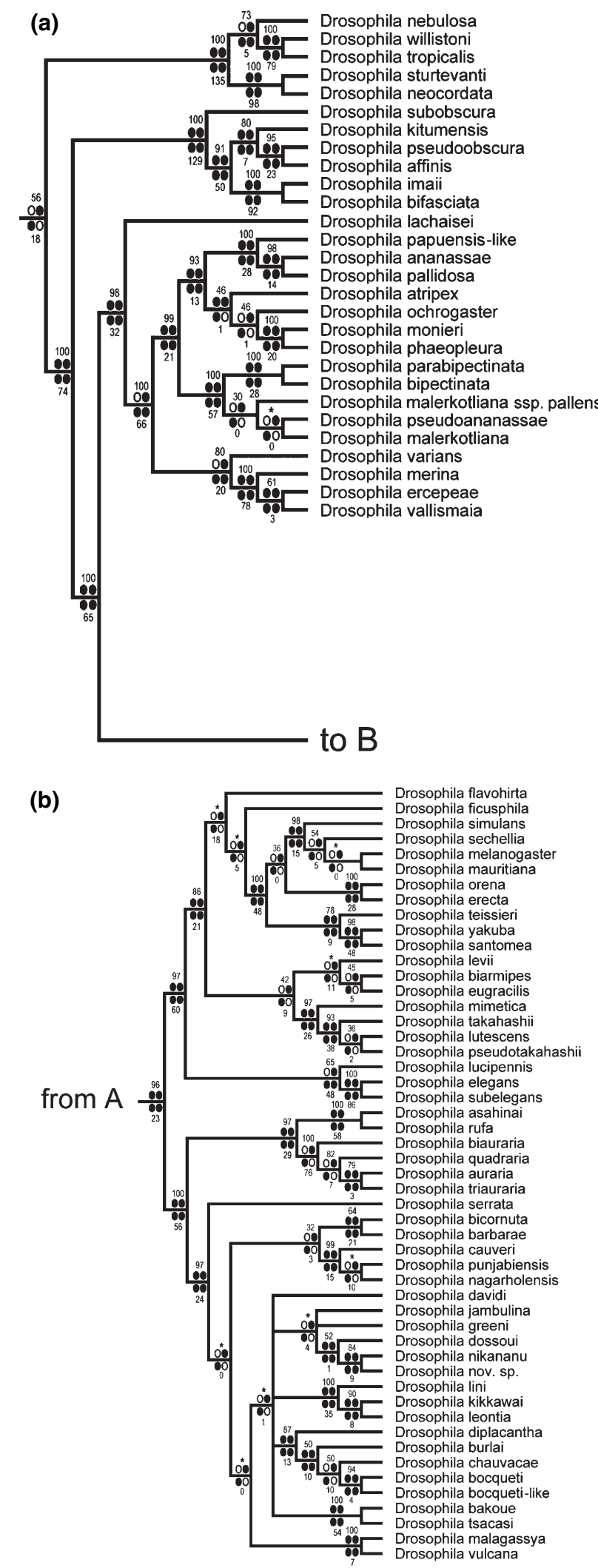

position is of little importance. In the following sections, the topologies of the different clades established by the different methods separately are presented.

\section{The subgenus Sophophora}

The following species groups represented in this study are usually considered to be the members of the subgenus Sophophora: melanogaster, obscura, saltans and willistoni. Although some of the reconstruction methods (i.e. MP uw, $\mathrm{NJ})$ suggest a very basal position for the Neotropical species groups willistoni and saltans, weighted MP, BI and ML analyses suggest a less basal position in which these two related species groups are clustered with other members of the subgenus Sophophora (thus forming clade 1). The remaining species groups, i.e. species groups melanogaster and obscura, form a monophyletic cluster. The nucleotide composition of Amyrel in the Neotropical species (Fig. 2), with an unusually low GC content, is likely to be responsible for the alternative placement of the species groups willistoni and saltans in both MP uw and NJ analyses. The latter hypothesis is well corroborated by the fact that a monophyletic subgenus Sophophora is recovered when the third codon positions are downweighted in MP analyses.

\section{The Neotropical species groups}

Regardless of their position in the global tree, the relationships within the species groups willistoni and saltans vary slightly depending on the method used. Drosophila willistoni and Drosophila tropicalis, from the species group willistoni, and D. sturtevanti and Drosophila neocordata, from the species group saltans, are invariably grouped together, whereas the position of D. nebulosa, a member of the species group willistoni, is variable in this analyses. Weighted MP analyses cluster the latter species with the willistoni-tropicalis pair of species. Other methods show $D$. nebulosa in a more basal position, with a moderate-to-high support: bootstrap values of 71\%(ML), 75\% (NJ-K2P), 66\% (MP uw) and posterior probabilities of 98\% (BI). Interestingly, the GC3\% of D. nebulosa is clearly higher $(56.3 \%)$ than the GC3\% of the other four species $(42.9 \%$ on average). It is also worth mentioning that the NJ-LD method, which is less sensitive to compositional bias, regroups D. nebulosa with the species group willistoni.

\section{The obscura species group}

In the data set pertaining to the study, the obscura species group appears to be the closest relative of the melanogaster

Fig. 4. Excerpt (corresponding to the clade 1 in Fig. 3) of the $50 \%$ majority-rule consensus tree (37 most equiparsimonious trees of 20607 steps; $\mathrm{CI}=0.21, \mathrm{RI}=0.76$ ) from the $4: 4: 1$ weighted parsimony analysis (heuristic search of 1000 random addition replicates). First and second codon positions were given a weight of four, whereas third positions were given a weight of one. The recovery of each node using four alternative weighting parameters is also given by two rows of circles (open circles figure the nodes that were not recovered under a specific weighting scheme). The top row corresponds to the results of the unweighted analysis (left) and the $2: 2: 1$ weighted analysis (right). The bottom row corresponds to the results of the $10: 10: 1$ weighted analysis (left) and the transition versus transversion weighted analysis (right). Numbers above the circle rows are bootstrap support values, whereas numbers below circle rows are Bremer support values (both bootstrap and Bremer support values were estimated under a $4: 4: 1$ weighting scheme). The nodes that were not recovered by bootstrap replicates are indicated by an asterisk. In addition, to improve the clarity of the figure, the recovery and the support values of some terminal nodes (indicated by labels) are shown at the bottom of the tree

(C) 2007 The Authors J Zool Syst Evol Res (2007) 45(1), 47-63 Journal compilation (C) 2007 Blackwell Verlag, Berlin 


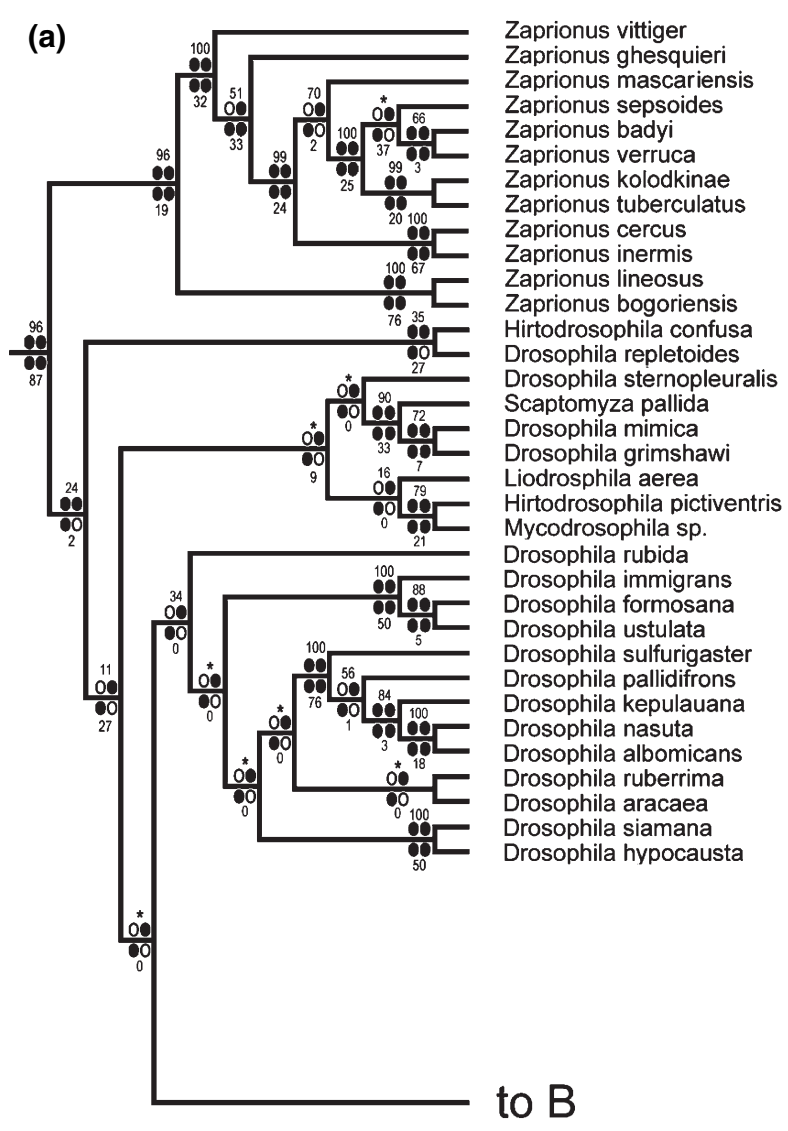

(b)

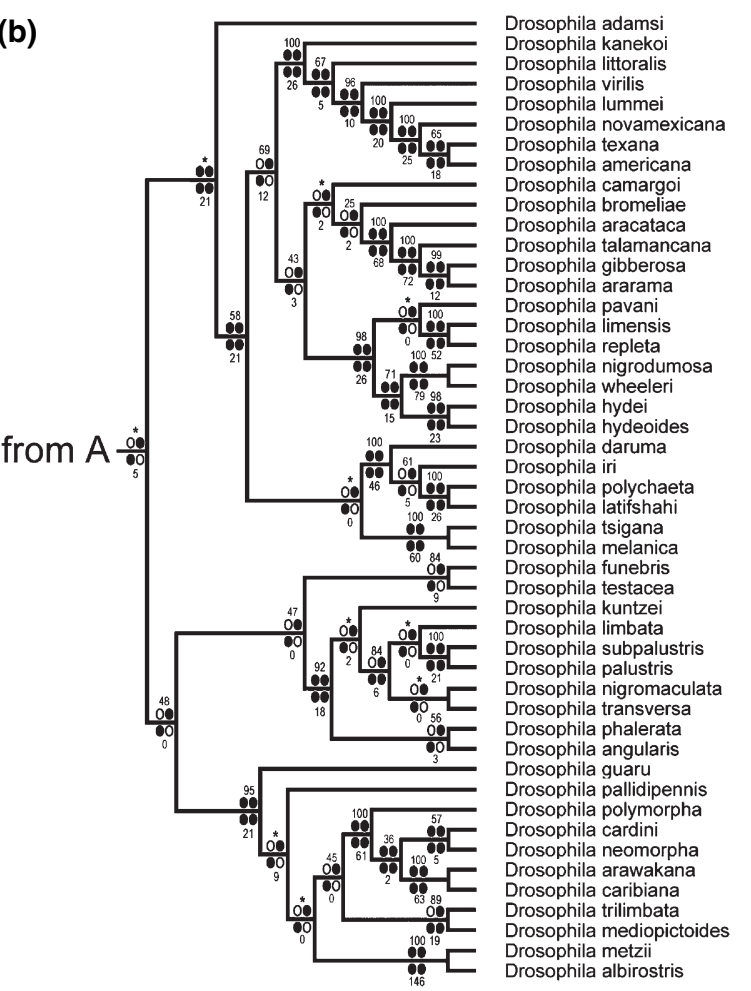

Fig. 5. Excerpt (corresponding to the clade 2 in Fig. 3.) of the $50 \%$ majority-rule consensus tree (37 most equiparsimonious trees of 20607 steps; $\mathrm{CI}=0.21, \mathrm{RI}=0.76$ ) from the $4: 4: 1$ weighted parsimony analysis (heuristic search of 1000 replicates of 100 random addition replicates each). Legend is as in Fig. 4

(C) 2007 The Authors J Zool Syst Evol Res (2007) 45(1), 47-63 Journal compilation @ 2007 Blackwell Verlag, Berlin species group. The topologies for this species group were similar for all the tree-building methods, with high support.

\section{The Drosophila melanogaster species group}

The D. melanogaster species group consists of 174 species (Bock and Wheeler 1972; Bock 1980; Lemeunier et al. 1986; Toda 1991). Twelve species subgroups have been described: melanogaster, montium, ananassae, takahashii, suzukii, eugracilis, ficusphila, elegans, rhopaloa, denticulata, flavohirta and longissima. Sixty-eight species or subspecies were studied: 30 from the montium subgroup ( 88 species described); all 9 known species in the melanogaster subgroup; 17 from the ananassae subgroup and several species from the so-called 'Oriental subgroups'-takahashii, suzukii, elegans, ficusphila, eugracilis and additionally, Drosophila flavohirta. All the reconstruction methods clearly show three main lineages with nested positions: (i) melanogaster + Oriental subgroups; (ii) montium subgroup; (iii) ananassae subgroup.

The melanogaster subgroup is well supported and is clustered with the Oriental subgroups. Within the melanogaster subgroup, the melanogaster complex is clearly apparent with D. melanogaster generally branching off from the triplet of species mauritiana-simulans-sechellia (best bootstrap support value of $87 \%$ in $\mathrm{NJ}-\mathrm{K} 2 \mathrm{P}$ ), but not in weighted MP reconstructions, which result in a clearly unusual topology for the subgroup, in which Drosophila simulans is further from mauritiana + sechellia than D. melanogaster (Fig. 4). BI, MP uw and Ts/Tv, ML and NJ methods suggest a cluster mauritiana-simulans, but with a low support. The other part of the melanogaster subgroup shows another two clusters $($ erecta + orena $)$ and $(y a k u b a+$ teissieri + santomea $)$. Drosophila santomea is sister to yakuba, as already shown with Amy + Amyrel (Cariou et al. 2001). Interestingly, these two clusters are found grouped together by several methods: BI and ML with high support; NJ, MP uw and Ts/Tv with an average support. Once again, MP $4: 4: 1,2: 2: 1$ and $10: 10: 1$ show an inconsistent result, as the yakuba complex is found to branch off first. It is noteworthy that if the melanogaster and yakuba species complexes are reliably based on consistent morphological and molecular evidence, the erecta + orena grouping is anything but a deep-rooted clustering. Although appearing as sister species in the tree reconstructions, these two species actually differ markedly from one another with regard to their morphology, and it has previously been suggested that they should not be included formally into a species complex sensu stricto (Lachaise and Silvain 2004).

The relationships of the originally Afrotropical melanogaster subgroup with the Oriental subgroups, and between these subgroups, remain unclear. Their hierarchical position depends on the reconstruction methods used. In all the methods, D. lucipennis diverges first from the clade (melanogaster subgroup + Oriental subgroups), and in all methods apart from MP uw, this species is clustered with the elegans subgroup, with high support (over $75 \%$ ). Then Drosophila ficusphila diverges in most methods. The relationships within the ingroup are less congruent between the different trees. However, the suzukii subgroup clearly appears to be polyphyletic, with Drosophila mimetica strongly attached to the takahashii subgroup, then to Drosophila biarmipes, whereas D. lucipennis, another member assigned to the suzukii subgroup, exhibits a close relationship with the elegans subgroup 
(a)
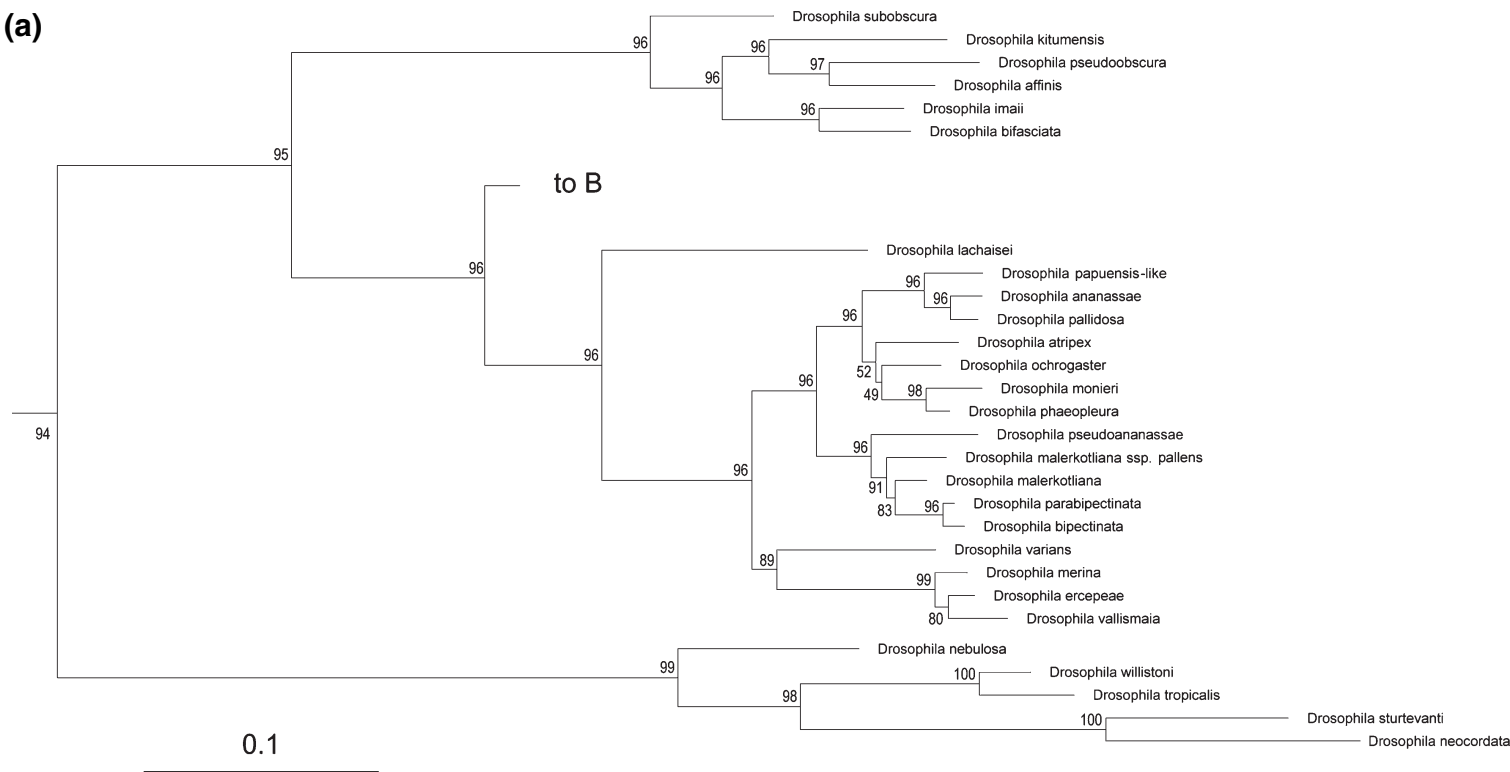

(b)

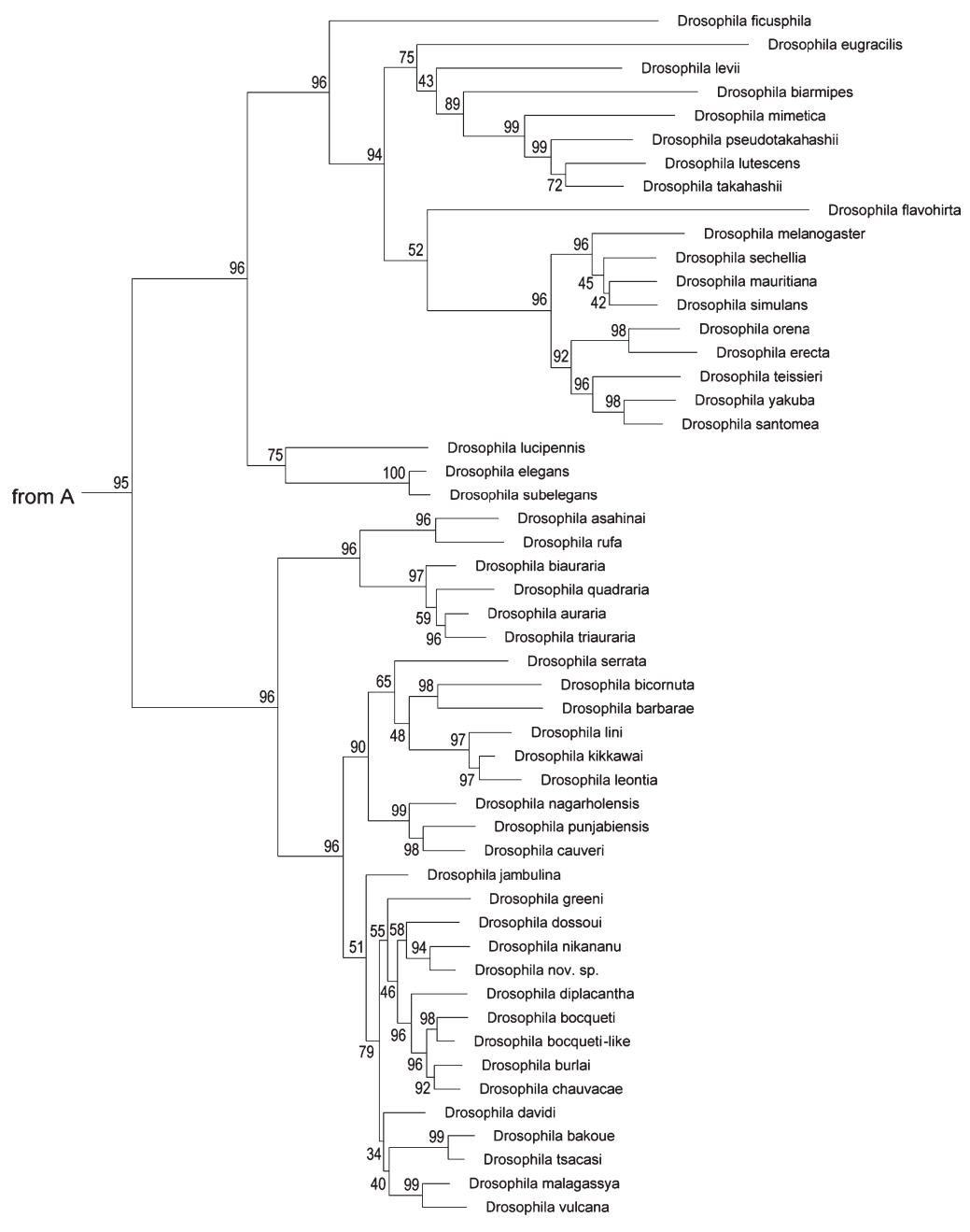

0.1

Fig. 6. Excerpt (corresponding to clade 1 in Fig. 3.) of the $50 \%$ majority-rule consensus tree from the Bayesian inference analysis ( 2000000 generations). Identical topologies were recovered in four distinct runs of MrBayes. Numbers at the nodes indicate the clade posterior probability estimates for each node 

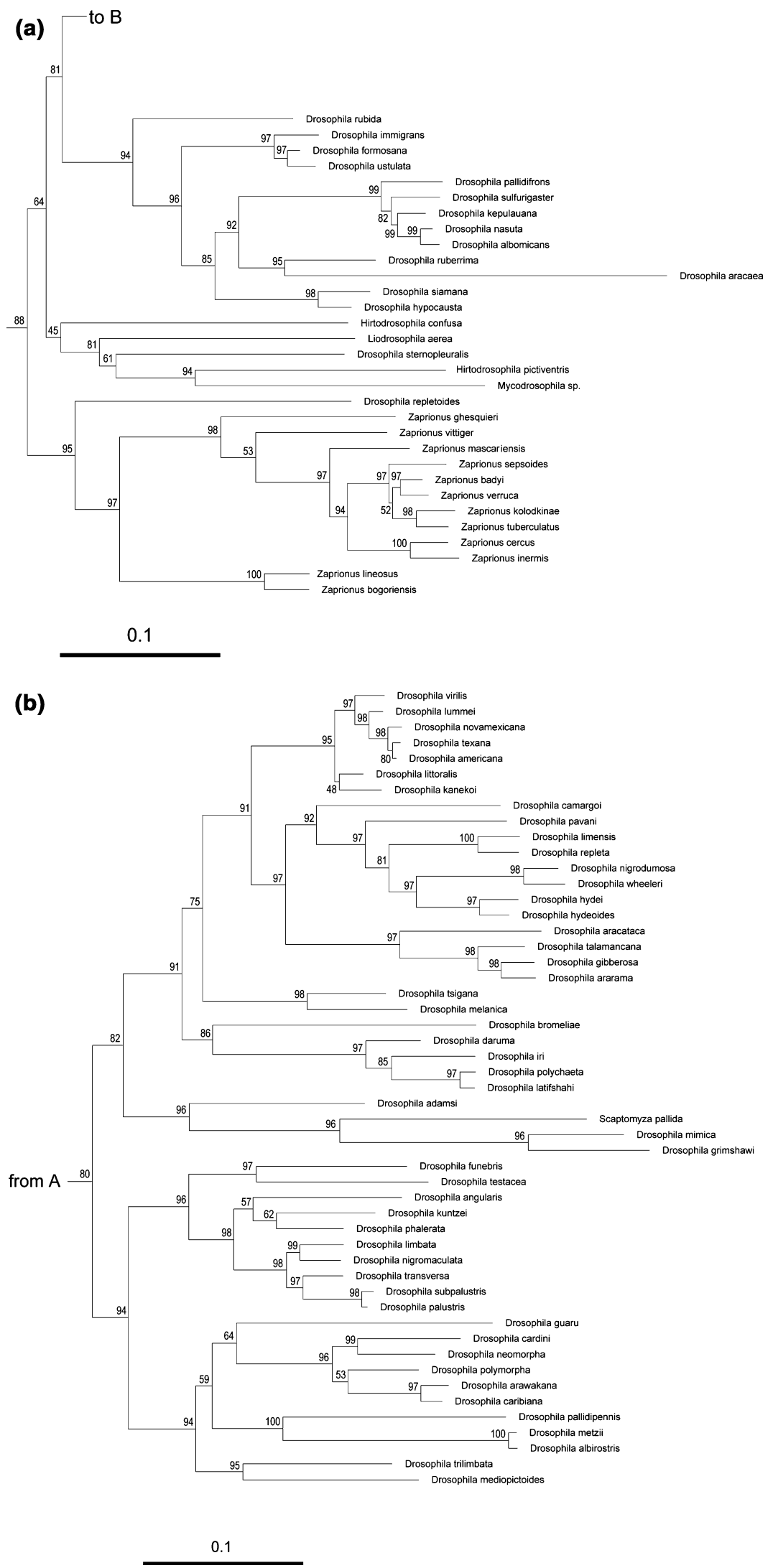

Fig. 7. Excerpt (corresponding to clade 2 in Fig. 3.) of the $50 \%$ majority-rule consensus tree from the Bayesian inference analysis (2 000000 generations). Identical topologies were recovered in four distinct runs of MrBayes. Legend is as in Fig. 6 
with high confidence, as already mentioned. In addition, the monophyly of the ficusphila subgroup (D. ficusphila, Drosophila levii) is not supported. Drosophila flavohirta clearly falls inside the melanogaster subgroup-Oriental subgroup clade, but its exact position is unclear, as is that of D. levii. Accordingly, the species or species subgroup closest to the melanogaster subgroup is not clearly defined.

\section{The montium subgroup}

The montium subgroup invariably appears monophyletic. Two main lineages emerge from this major tropical subgroup: an auraria lineage and a kikkawai lineage. The auraria lineage is comprised of Drosophila asahinai and Drosophila rufa, clustered together and, basal to the (Drosophila auraria, Drosophila biauraria, Drosophila triauraria, Drosophila quadraria) cluster, of which D. auraria and triauraria are always grouped together. The kikkawai lineage includes the other species. In all reconstructions, the following species were clearly clustered together: (bicornuta, barbarae), (punjabiensis, cauveri, nagarholensis), serrata, (kikkawai, lini, leontia). Another cluster, less well supported, groups the remaining species of the montium subgroup, with Drosophila jambulina diverging first, except for weighted MP. Within this cluster, an Afrotropical unit, comprising bocqueti, bocqueti-like, burlai, chauvacae, diplacantha, displays stable topology. The position of a few species, Drosophila serrata, Drosophila davidi, Drosophila vulcana and Drosophila greeni, appears very sensitive to the phylogenetic method used. The new species from Príncipe Island in the Gulf of Guinea, assumed to be close to Drosophila nikananu on the basis of its morphology, is confirmed here to be nikananu-like.

\section{The ananassae subgroup}

The ananassae subgroup consists of ca. 22 species and at least 2 subspecies (Tobari 1993; Lemeunier et al. 1997), divided into three species complexes - ananassae, bipectinata and ercepeae, and a few ungrouped species. Seventeen taxa were available in this study. All methods give essentially the same topology, with the three complexes well supported and hierarchized. Interestingly, Drosophila varians and Drosophila lachaisei, which were once included in the ananassae complex (Lemeunier et al. 1986) are in fact found to be the most divergent species. Drosophila lachaisei is always basal to the subgroup. The branching of $D$. varians with the ercepeae complex is not well supported in MP nw but is highly supported when data are weighted; the BI also shows a high posterior probability value. Drosophila varians is proposed to be more basal by both ML (with $86 \%$ value) and $\mathrm{NJ}(64 \%)$ but is still ingroup relative to D. lachaisei. The bipectinata complex consists of closely related species, which may give rise to interspecific hybrids (Bock 1978). Drosophila pseudoananassae, which emerges basal in this complex (except by weighted MP), is divided into two subspecies $-D$. p. pseudoananassae and D. p. nigrens, which differ by their mitotic karyotype (Lemeunier et al. 1986). In this study, it was found that their Amyrel sequences were identical (only one is shown in the trees). Perhaps, shared ancestral polymorphism is obscuring the relationships between Drosophila malerkotliana, Drosophila bipectinata and Drosophila parabipectinata (not shown, but see the position of the subspecies malerkotliana pallens). Drosophila pallidosa is confirmed by almost all the trees as the sister species of Drosophila ananassae, and is in fact the only species to produce viable hybrids with D. ananassae (Bock 1984). However, ML suggests that there may be a relationship between $D$. pallidosa and Drosophila papuensis-like.

\section{The subgenus Drosophila and related genera}

A common root for the subgenus Drosophila and distinct genera is found in several trees, with medium-to-high support (97\% and BS of 60 in MP $4: 4: 1,88 \%$ in BI, 63\% in NJ-LD, $58 \%$ in MP $2: 2: 1$ ). Some taxa differing markedly morphologically, and considered to be distinct genera are always positioned inside the subgenus: Zaprionus, Scaptomyza, Hirtodrosophila, Liodrosophila, Mycodrosophila, thus making the subgenus Drosophila paraphyletic. However, within this large cluster, the relative positions of species groups or other genera are poorly defined, although some species groups are well supported.

\section{The well-supported clades}

The genus Zaprionus forms a monophyletic cluster, with the subgenus Anaprionus clearly differentiated. Zaprionus (Anaprionus) lineosus harbours a 13-bp deletion in the coding sequence, resulting in a frameshift. This has been confirmed by two independent amplifications on two individuals from the same stock. The relationships between this genus and other groups of Drosophilidae are not clearly resolved by all the methods. It is clearly inside clade 2 , and is basal to this clade in most MP trees and by the BI method.

The immigrans species group is well supported in all methods and is monophyletic. However, species members of the two taxonomic subgroups hypocausta and immigrans are scattered within the immigrans clade. Drosophila rubida (a member of the hypocausta subgroup) diverges first and appears very distant from the two other species of the hypocausta subgroup, Drosophila siamana and Drosophila hypocausta. Similarly, in the immigrans subgroup, Drosophila ruberrima is not clustered with the rest of its subgroup, but with the nasuta subgroup. The different reconstruction methods are inconsistent with regard to the clustering of other species groups with the immigrans group. Both the BI and weighted MP analyses isolated the immigrans group in a basal position, whereas the NJ methods and MP-Ts/Tv cluster this group with a wellsupported (tripunctata, quinaria, funebris, testacea, guarani, cardini) clade, but with low support. Drosophila trilimbata, which belongs to the immigrans group, is clustered with the cardini group or with Drosophila mediopictoides (tripunctata group), which is itself clustered with the cardini group. The cardini group is connected to the tripunctata group, to D. pallidipennis and to Drosophila guaru (guarani group). The bootstrap values for this clade range from $54 \%$ (MP uw) to $95 \%$ and a BS of 21 is recovered (MP $4: 4: 1$ ). Drosophila mediopictoides is separated from Drosophila metzii and Drosophila albirostris, suggesting a paraphyletic status of the tripunctata group. The quinaria group is clearly identified and is clustered with the testacea and funebris groups. Interestingly, this clade harbours, in each species group, a number of mushroom-feeding species abundant in temperate and boreal forest (Prigent et al. 2003). In MP $4: 4: 1$, the quinariafunebris-testacea clade is clustered with the cardini clade with a low support ( $48 \%$ bootstrap and null BS) but is also found by the BI tree, with a $94 \%$ posterior probability.

(C) 2007 The Authors J Zool Syst Evol Res (2007) 45(1), 47-63 Journal compilation (C) 2007 Blackwell Verlag, Berlin 
Another clade, well supported by most methods, contains the following groups: virilis, repleta, annulimana, mesophragmatica, dreyfusi, melanica, polychaeta and bromeliae. MP 4:4:1, MP 2:2:1, ML, NJ and BI show polychaeta branching off first (the results from MP uw and ML have been excluded, according to which the polychaeta group clusters with the subgenus Sophophora). The melanica group diverges from a (virilis + annulimana + mesophragmatica + repleta + dreyfusi) cluster in BI and NJ. In contrast, MP $4: 4: 1$, MP $2: 2: 1$ and MP $10: 10: 1$ suggest that melanica is clustered with polychaeta. NJ-K2P indicates a virilis-annulimana clade (bootstrap support of $89 \%$ ) connected to a dreyfusi-repleta clade, whereas MP $4: 4: 1$, MP $2: 2: 1$, MP $10: 10: 1$ and $\mathrm{BI}$ tended to suggest that annulimana diverges from repleta, with camargoi (dreyfusi group) linked to either repleta or annulimana, and that the virilis group branches off from this triad. The position of Drosophila bromeliae is not clear: NJ trees show that this species are branching off just above melanica, whereas BI suggests a connection to polychaeta and MP a connection to annulimana. It should be noted that Drosophila pavani, a member of the mesophragmatica group, is found either inside or outside the repleta species group.

\section{The other groups or genera}

There is no clear resolution for positioning the other taxa studied here, some of which being ungrouped by taxonomists (Bächli 1999-2005). Scaptomyza pallida is clearly closely related to the Hawaiian Drosophila mimica and Drosophila grimshawi, but the position of this cluster is uncertain. It may either be close to Drosophila adamsi and connected to the virilis-repleta clade (under BI) or occupy an external position in the subgenus Drosophila (MP, but low support). The genus Hirtodrosophila is represented in this study by $H$. confusa and Hirtodrosophila pictiventris. These two species are not obviously connected to each other. In fact, $H$. pictiventris is closer to the Mycodrosophila sample (unidentified species). Liodrosophila aerea is branched with Mycodrosophila $+H$. pictiventris in weighted MP and BI trees, with low support in MP, and with Drosophila sternopleuralis (histrio group) in ML and NJ. Indeed, the support is invariably low. Drosophila repletoides (tumiditarsus group) is branched with $H$. confusa in MP, with Zaprionus in NJ, BI and ML. Drosophila adamsi is basal to the 'supergroup' virilis in MP, and also in the BI method, but in this case linked to the Hawaiian flies. The other methods also show this species to be linked to the Hawaiian-Sc. pallida clade. Drosophila aracea is shown to be a member of the immigrans group in the BI tree, with a very long branch. This species is similarly located in the MP tree (weighted reconstructions only). Alternatively, this species is found with the quinaria group and Mycodrosophila in NJ trees. Another ungrouped species, Drosophila pruinosa, has not been included in the trees presented here. However, preliminary results (NJ, MP) suggest a clustering with $D$. sternopleuralis.

\section{Discussion}

Using several reconstruction methods, and for most of them, incongruences or weaknesses at some levels have been revealed. However, it appeared that both weighted MP (with downweighting of the third codon positions) and BI analyses were more satisfactory for dealing with base composition bias and gave more robust trees. Overall, it appears that the use of weighting schemes (especially through the downweighting of the third codon position) provides results that match the morphological data for deeper nodes better, whereas unweighted analyses perform better for terminal nodes. The loss of phylogenetic information resulting from the downweighting of the third codon position probably accounts for the latter observation and this highlights the difficulty of defining optimal parsimony weighting schemes. In contrast, the results of the BI analyses appear to be more homogeneous, yielding well-supported results at all levels of the tree. This could probably be attributed to the supposedly better performance of likelihood-based methods when one analyses data sets exhibiting significant base composition heterogeneity between taxa (Galtier and Gouy 1995; Galtier and Gouy 1998).

The general topology of the phylogeny, rooted with L. maculata, a Drosophilidae (Steganinae) and a much less closely related species, the Tephritidae $C$. capitata, always shows Scaptodrosophila and Chymomyza, as distinct genera. Two clades constitute the genus 'Drosophila', which is paraphyletic according to the current classification. The first clade corresponds to the subgenus Sophophora, which appears to be monophyletic; however, species from the Lordiphosa genus was not included, which was shown itself to be polyphyletic (Katoh et al. 2000). Indeed, earlier studies have suggested that some Lordiphosa species could be related to Sophophora as a sister clade to the Neotropical groups willistoni and saltans (Katoh et al. 2000). The second clade (roughly corresponding to the subgenus Drosophila) is clearly paraphyletic, as demonstrated by many investigators since Throckmorton (1975). As a consequence, there is ever-growing evidence, including the present data set, which calls into question both the genus and subgenus Drosophila. However, it is merely pointed out in passing the need to revisit the classification of Drosophilidae above the species group level and focus here solely on the species group level.

\section{Questioning the traditional melanogaster species group}

On the basis of the data presented in this study, it is proposed to question the traditional boundaries of the melanogaster species group, which is a quantitatively important taxonomical unit in the subgenus Sophophora. However, this species group is such a mix of contrasted morphological patterns that it has long been seen as a confused taxonomical unit. The historical vicissitudes of the definition of the melanogaster group, as new species have accumulated (discussed in Bock and Wheeler 1972, pp 1-8; Lemeunier et al. 1986, pp 1156-157), reflects the difficulties met with in such an extended species group.

The concepts of species groups and subgroups are nearly always used in relation to Drosophilidae taxonomy, and they are not commonly used in other insect families. As a result, they are of only relative practical value. Can these taxonomical levels be recognized independently of evidence of phylogenetic relatedness? How old would have a monophyletic cluster to be, to be called a 'species group'? Should the size of the cluster in the definition be considered? In fact, no rule and no definition exist and this is certainly one of the weaknesses of the Drosophilidae taxonomical classification, and more especially for all the hierarchical levels between species and genus.

The traditional melanogaster species group is a questionable taxon, and the question raised here is: Is such a large and composite taxonomical unit needed? The melanogaster species group does not suggest any clearly identifiable diagnostic 
pattern. Although a definition of the melanogaster group has been proposed by Bock and Wheeler (1972), and refined in Lemeunier et al. (1986) by including functional characteristics of the male genitalia, it includes a number of characteristics that do not apply to all species implicated. In contrast, all taxonomists have long accepted a clear immediate and empirical picture of the montium-like, ananassae-like and melanogaster (sensu stricto)-like morphological patterns. From the earliest days of Drosophila classification (Hsu 1949), the melanogaster group was not given any definition but species subgroups, notably the ananassae, melanogaster and montium subgroups imposed themselves as clear-cut homogeneous morphological units. In view of the clear data from the Amyrel-based phylogenetic reconstruction obtained here, which is consistent with other data in the literature (Goto and Kimura 2001; Kastanis et al. 2003), it is proposed to elevate both the ananassae and montium species subgroups to the rank of species group. An updated definition of the neo ananassae, melanogaster and montium species groups will be given elsewhere (Lachaise et al. in preparation). Henceforth, in the discussion, a new terminology is used, in which the ananassae and montium species subgroups are viewed as species groups, and the melanogaster species group is considerably reduced to include only melanogaster and the 'Oriental' species subgroups, the status of which remains unchanged.

\section{The ananassae species group, new status}

The ananassae species group is well sampled in this study. Its location outside the montium-(melanogaster + Oriental) clade is clear, with a $100 \%$ support in all methods. This is consistent with other studies: Goto and Kimura (2001), Kastanis et al. (2003), Lewis et al. (2005). Tamura et al. (2004) have estimated divergence times between Drosophila species and suggest a longer divergence time between $D$. melanogaster and $D$. ananassae than between $D$. melanogaster and the montium species group. Other studies have suggested various branching topologies, but the supports are generally low in these cases: The ananassae group is sister to the Drosophila obscura group in Pélandakis and Solignac (1993), sister to montium in Schawaroch (2002) and sister to the melanogaster subgroup in Yang et al. (2004), with montium located further outside. Within the D. ananassae group, the three taxonomic complexes are clearly isolated and nested. New data concern the position of the ungrouped species $D$. varians and D. lachaisei. The case of the latter species is very interesting in view of the history of this species group, which is considered to be of Oriental origin (Lemeunier et al. 1986). Drosophila lachaisei has been found as a rare species (a total of only some two dozen individuals have been caught) from Western, Central and Eastern Africa. The fly used in this work came from São Tomé Island. Interestingly, D. lachaisei, which is typically a Palaeoendemic species with a broad fragmented and scattered historical home range, is basal to the entire subgroup, suggesting a very ancient colonization of the Afrotropical region by a member of the ananassae group, of which this species is probably a relic.

\section{The montium species group, new status}

The montium species subgroup has so far been viewed as the most species-rich taxonomical unit within the melanogaster species group, and one third of the known species were studied here. The data unequivocally show that it forms a well-defined monophyletic cluster with strong support, thus confirming the recent work of Schawaroch (2002) and Zhang et al. (2003). In view of this consistency plus the marked morphological similarity, and despite its wide geographic distribution covering the Oriental, Afrotropical, Australasian and East-Palaearctic regions, it is suggested that the montium subgroup should be raised to species group rank. Hereafter, this will be referred to as the montium species group.

The montium species group is clearly sister to the melanogaster subgroup + Oriental subgroups. This result conflicts with the results reported by Schawaroch (2002), who identified the montium lineage as a sister (albeit with weak statistical support) of the ananassae lineage, and with the unlikely trichotomy D. lucipennis (suzukii subgroup)-montium subgroup-elegans subgroup (Lewis et al. 2005), but it is fully consistent with previous studies (Bock 1980; Ashburner et al. 1984) and with most gene data (Pélandakis and Solignac 1993; Inomata et al. 1997; Goto and Kimura 2001). The mitochondrial sequences were not taken into account, which are generally considered to be less reliable for phylogenetic reconstructions in the genus Drosophila (discussed e.g. in Goto and Kimura 2001).

Separating the montium group from other species groups is easy, whereas diagnostic identification is often critical within the montium species group because of a high level of withingroup similarity regarding both male terminalia and sex combs, and to morphological homoplasy (convergence). As a result, the relationships between species and taxonomic complexes are particularly unclear. Amyrel provides valuable information by identifying some well-supported groupings consistent with the classical taxonomy and reveals some taxonomic inconsistencies. Data on Amyrel, like previous molecular analyses (Schawaroch 2002; Zhang et al. 2003), do not for instance support the Afrotropical D. greeni as a member of the bakoue complex (Rafael 1984; Lemeunier et al. 1986). In addition, the Afrotropical Drosophila diplacantha initially ascribed to the kikkawai complex (Tsacas and David 1977) clearly belongs to the bocqueti complex (sister of the nikananu complex), a position that was previously indicated by Amy genes (Zhang et al. 2003), the morphological affinity with the kikkawai complex being a homoplasy. Furthermore, the results of this study support the hypothesis that sex comb morphology similarity between the nikananu complex and the melanogaster subgroup also results from convergence, as proposed by Schawaroch (2002) rather than being indicative of an evolutionary link between the montium and melanogaster subgroups (Tsacas and Chassagnard 1992).

Compared with previous analyses, a much clearer picture of the evolutionary history of the montium group seems to emerge from the present study. The primeval split between a strictly Oriental auraria-rufa lineage and an Oriental-Afrotropical lineage (from D. serrata to D. vulcana in Figs 4 and 6), coupled with the present geographic distribution of the species, support the Oriental origin of the species group. This conclusion is consistent with the two most comprehensive studies in terms of taxon sampling, that is those of Schawaroch (2002) and Zhang et al. (2003). In these studies, based on the nuclear Amy, Adh and $h b$ genes and the mitochondrial COII, the clade that includes auraria-rufa was basal, although a few Asian species, namely Drosophila kanapiae and Drosophila parvula, which were not included in the present study, were located outside.

The reconstruction shown in Fig. 6 suggests a second-level split within the montium species group separating the primarily

(C) 2007 The Authors J Zool Syst Evol Res (2007) 45(1), 47-63 Journal compilation ( 2007 Blackwell Verlag, Berlin 
Oriental kikkawai-nagarholensis-barbarae lineage and the Afrotropical greeni-nikananu-bocqueti-bakoue lineage. The former lineage is actually comprised of Oriental species and species that have wider geographical distribution, such as the Australasian D. serrata or the circumtropical Drosophila kikkawai, the geographical range of which partially overlaps the Palaearctic region. The second lineage consists exclusively of taxa from the Afrotropical region, and it is therefore postulated that all the Afrotropical species of the montium group have a common origin. According to the constructions used (not shown), either D. greeni or the pair Drosophila vulcana-Drosophila malagassya might be the most basal species, which agrees with the Amy genes (Zhang et al. 2003). The position of $D$. jambulina, a species widely distributed from Taiwan to India, invariably clustered with the Afrotropical lineage, is interesting in that it appears to be a possible link to the Oriental species within this highly diversified Drosophila species group which has undergone extensive geographic dispersal.

\section{The melanogaster species group, new status}

Once the ananassae and montium clades have been removed from the large traditional melanogaster species group and raised to their own species group status, the remaining species subgroups, namely the melanogaster and the 'Oriental' species subgroups become the only two components of the novel restricted melanogaster species group.

Classically, the melanogaster species subgroup is divided into three complexes, nested as [erecta(yakuba(melanogaster))] (Lachaise et al. 1988). The data do not solve the problem of the trifurcation of the triad D. simulans, Drosophila mauritiana and Drosophila sechellia, inside the melanogaster complex, which reflects speciation events that took place within a short period (Kliman et al. 2000). The support values are low, and the topologies vary depending on the reconstruction method. The main point deserving discussion here is the clustering of the yakuba species complex with the erecta-orena clade, rather than with the melanogaster species complex. This is proposed by most methods applied to Amyrel. Although this conflicts with the classical view that the erecta-orena clade diverged first [Cariou 1987; Lachaise et al. 2004; but see the two possibilities in Lachaise et al. 1988, and note that when Drosophila orena was used as an outgroup in both Amyrel and Amy phylogenetic trees (Cariou et al. 2001), the topology reflected the classical view], it is consistent with molecular phylogenies obtained using other markers. While the $A d h$-based phylogenies of Russo et al. (1995), Katoh et al. (2000) (minimum evolution method for the latter) and Lachaise et al. (2000) (period and allozymes) agree with the classical view with good support, the trees proposed by Kastanis et al. (2003) on mtDNA, Ko et al. (2003) on $A d h+A d h r+G l d+X d h$, Tatarenkov et al. (1999) on $D d c$, Katoh et al. (2000) on $A d h$ (with MP method), Lewis et al. (2005) on COI + COII show a clade (erectaorena $)+($ yakuba-santomea-teissieri) with good support values. In a recent review (Lachaise and Silvain 2004), it was argued that this latter five-species clade includes three species, either strictly endemic (D. santomea and D. orena) or partly endemic $(D$. erecta) confined to the Cameroon volcanic line. Although the two other allied species (Drosophila teissieri and Drosophila yakuba) have extended their distribution to Eastern and South-eastern Africa since their speciation, they were also thought to have originated at different times from that mountain region, the uplift of which dates back some 13-15 Myr ago. A clear-cut split between a monophyletic Western clade (erecta-orena $)+($ yakuba-santomea-teissieri $)$ and a monophyletic Eastern clade (melanogaster) + (simulans, sechellia, mauritiana) would satisfactorily match the palaeobiogeographic pattern proposed in the aforementioned paper. In particular, this would provide further support for the nascent hypothesis of a major West-East separation of the African mainland that might predate the formation of the Rift.

Turning to the ever-puzzling question of the affinities of the Oriental species subgroups, the findings of this study do not clarify which of the Oriental subgroups is the closest to the melanogaster subgroup. The relationships between the Oriental subgroups are not well solved, except that Drosophila elegans does indeed seem to have diverged first, as already proposed by Goto and Kimura (2001). As shown by earlier studies (Pélandakis and Solignac 1993; Goto and Kimura 2001; Kopp and True 2002; Schawaroch 2002; Lewis et al. 2005), the suzukii subgroup is polyphyletic. Some species are related to the takahashii subgroup, and $D$. lucipennis is close to D. elegans. Drosophila flavohirta, which was previously ungrouped within the traditional D. melanogaster group, is clearly included in the Oriental cluster, although not clearly related to any other species. Several methods propose D. flavohirta as the closest relative of the melanogaster subgroup, although with low support.

\section{Difficulties with the Neotropical groups and biased composition}

The conflictual position of the Neotropical groups in the reconstructions has been highlighted earlier, with their widely accepted membership to the subgenus Sophophora (O'Grady et al. 1998; O'Grady and Kidwell 2002). The particularly low GC content of Amyrel in these species has been pointed out. This unusual compositional bias has been reported for several genes in species of the willistoni and saltans groups. It has been shown that it is a derived state and that the ancestral base composition in Drosophila had a high GC content (RodriguezTrelles et al. 2000). It has been suggested that this conflicting base composition was mainly responsible for the misplacing of the Neotropical groups in the phylogeny. Indeed, the weighted MP methods, which lower the influence of $\mathrm{GC} 3 \%$, give a clustering at the base of the subgenus Sophophora. This problem has been addressed by Tarrio et al. (2001), using sophisticated models (Galtier and Gouy 1998) accounting for the non-uniformity and non-stationarity of substitutions. The results clearly showed the branching of the Neotropical groups basal to Sophophora, with high bootstrap values. This has also been found by other workers (Russo et al. 1995; Kwiatowski and Ayala 1999; Tatarenkov et al. 1999), albeit with lower support. However, Tatarenkov et al. (1999) obtained good support when using only codon positions $1+2$. Katoh et al. (2000) placed these groups with Sophophora (with low support) using parsimony methods, but outside the Drosophila genus using minimum evolution method.

\section{The deep nodes in the subgenus Drosophila are not well resolved}

Interestingly, for the external branches of the tree, Tarrio et al. (2001) had found that using C. capitata as the outgroup, the first diverging taxon was Scaptodrosophila, followed by Chymomyza, as was found with MP (Fig. 3a). These authors have pointed out that $C$. capitata itself has a low GC content 
(Fig. 2), which could be detrimental to the reconstruction if not accounted for. Their data provide strong support for the possibility that the genera Zaprionus and Hirtodrosophila should be included in a clade involving the subgenus Drosophila, as was found $(96 \%$ bootstrap and BS of 87 in MP $4: 4: 1)$. The position of Zaprionus and of taxa considered to belong to distinct genera (Scaptomyza, Hirtodrosophila) within the 'subgenus' Drosophila has been consistently proposed earlier, among many others, notably by Throckmorton (1975), Pélandakis and Solignac (1993), Tatarenkov et al. (1999), Kwiatowski and Ayala (1999), and Katoh et al. (2000) and should now be accepted. As a consequence, the current classification in the subgenus Drosophila appears unquestionably paraphyletic suggesting that the generic and subgeneric classification must be revisited. The deep nodes within the current subgenus Drosophila are unfortunately not clearly resolved by the data, and this remains a shortcoming. Indeed, Tatarenkov et al. (1999), Katoh et al. (2000) and Remsen and O'Grady (2002), who investigated various species groups and closely related genera, found generally low support for the deep nodes. Kwiatowski and Ayala (1999), working with a limited number of species and using combined data from $A d h$ and $S o d$, found high support for a relationship between the Hawaiian-Scaptomyza and the virilis-repleta clade, on one hand, and between Hirtodrosophila and Drosophila immigrans, on the other hand. The branching of Hawaiian species with the virilis-repleta clade was also found by Amador and Juan (1999) using Adh. These conclusions could not be clearly confirmed. Data using only the same (or similar) species as those in Kwiatowski and Ayala (1999) in an MP $4: 4: 1$ assay (100 bootstraps of 100 heuristic replicates) and NJ-K2P or LD have been reanalysed. The relationships reported by these authors (not shown) were yet to be found. It is however worth pointing out that the Neotropical species groups were found strongly branched at the base of the subgenus Sophophora (bootstrap value of $90 \%$ with MP).

The topology of the virilis group is consistent with the phylogeny proposed by Spicer and Bell (2002). A relevant sampling for several of the species groups included in the virilis-repleta 'supergroup' [remembering the virilis-repleta radiation of Throckmorton (1975)] was had: six species for the repleta group, seven species for the virilis group, four species for the polychaeta group, four species for the annulimana group, two species for the melanica group, which may improve the reliability of the phylogeny (Zwickl and Hillis 2002). Several topologies are proposed by the different methods, but all of them place polychaeta as a basal group, followed by melanica. The hierarchy proposed by the BI tree in this study is the same as that shown by Robe et al. (2005), for the Amd gene. The MP 4:4:1 tree shows a topology similar to that proposed by Tatarenkov and Ayala (2001) from combined Amd and $D d c$ gene sequences. These authors also found a good support for placing $D$. bromeliae ingroup relative to the annulimana group, whereas none of the trees confidently shows branching of $D$. bromeliae. As discussed above, the Hawaiian species have been proposed to be the closest sister group to the virilis-repleta clade (e.g. Amador and Juan 1999; Tatarenkov and Ayala 2001), but no such significant branching was found in this study, with the noticeable exception of the BI tree. Only two Hawaiian species were included here, while the Hawaiian radiation gave hundreds of species. More species could help clarifying the real topology. More detailed studies within the Hawaiian species have been conducted
(Baker and DeSalle 1997; DeSalle and Brower 1997; Remsen and DeSalle 1998; Bonacum et al. 2005).

The immigrans group is clearly monophyletic, although some discrepancies regarding the classical taxonomy of two of the subgroups sampled here were found. It has been suggested that the immigrans group may be a member of a immigranstripunctata radiation, well separated from the virilis-repleta radiation, with the tripunctata group linked to quinaria, funebris and cardini (see Bächli 1999-2005). This view had been supported by previous studies (Pélandakis and Solignac 1993; Katoh et al. 2000; Remsen and O'Grady 2002); however, none of the trees supports this cluster with confidence. Although some methods, such as NJ, do suggest this classical view, the level of support is very low. The best trees show the immigrans group branching off first and the tripunctata clade tends to be linked to the virilis-repleta clade. The other groups mentioned are clearly clustered together by most methods. However, the hierarchy within this clade is not exactly the same as that found by other workers (Robe et al. 2005). Interestingly, we have also been found that the tripunctata group is probably paraphyletic, as has been shown by other authors (e.g. Robe et al. 2005).

The phylogenetic trees presented in this study are gene trees, constructed using a single gene, Amyrel. Interestingly, many results in this study are consistent with previous findings and phylogenetic analyses. In addition, the recent work by Kopp (2006) shows that Amyrel gives a useful phylogenetic information. This gives confidence regarding the analyses of this study reflecting the phylogeny of Drosophilidae rather than the evolution of a specific gene merely. It is likely that combined analyses from several additional genes would lead to more reliable phylogenies, but available sequences of other genes in the literature or databases correspond to only a limited fraction of the sampling in this study. The problem of using more taxa or more characters for improving phylogenies has been debated under several aspects (e.g. Zwickl and Hillis 2002; Hillis et al. 2003; Gontcharov et al. 2004; May-Collado and Agnarsson 2006). Delsuc et al. (2005) stated that 'A long-standing debate in phylogenetics is whether the greatest improvement in accuracy results from an increased number of characters (in this case, genes) or species. Evidence from computer simulations has been equivocal, whereas empirical studies tend to support the importance of extensive species sampling.' The phylogeny presented here may be therefore improved by additional taxa as well as addition of other genes. Finally, Amyrel, which allows easy amplification of the full coding sequence all at once, offers a powerful tool to investigate Drosophila phylogeny. It is hoped that Amyrel will be used by researchers attempting to clarify the phylogenetic relationships of their Drosophila species of interest, thus enlarging the trees proposed here.

\section{Acknowledgements}

We thank H. Hachani and S. Prébot for technical help, E. Renard for providing sequence data on the obscura species group and Kim van der Linde for fruitful discussion. The Gif laboratory collection is part of the Centre de ressources Drosophiles, granted from the CNRS and the Ministère de la Recherche ( $\left.\mathrm{n}^{\circ} 03 \mathrm{G} 138\right)$.

\section{Résumé \\ Une phylogénie des Drosophilidae avec le gène Amyrel: remise en question des limites du groupe d'espèces Drosophila melanogaster}

(C) 2007 The Authors J Zool Syst Evol Res (2007) 45(1), 47-63 Journal compilation (C) 2007 Blackwell Verlag, Berlin 
Cette étude analyse les relations phylogénétiques chez les Drosophilidae. Nous avons utilisé le gène Amyrel, un paralogue éloigné de la famille multigénique des alpha-amylases, sur 164 espèces, en essayant d'avoir un bon échantillon sur de nombreux groupes d'espèces de Drosophila et quelques genres voisins. Les séquences nucléiques ont été analysées en maximum de parcimonie, neighbor joining, et avec des méthodes probabilistes. Nous avons tenu compte de l'hétérogénéité de composition (surtout la faible teneur en GC dans les groupes willistoni et saltans) Nos résultats montrent que le genre Drosophila est paraphylétique; et si le sous-genre Sophophora apparaît monophylétique, le sous-genre Drosophila est paraphylétique, certains genres distincts y étant inclus. Nous proposons d'élever au rang de groupes d'espèces les sous-groupes ananassae et montium, et de limiter le groupe melanogaster au sousgroupe melanogaster et aux sous-groupes « orientaux».

\section{References}

Amador A, Juan E (1999) Nonfixed duplication containing the Adh gene and a truncated form of the Adhr gene in the Drosophila funebris species group: different modes of evolution of Adh relative to Adhr in Drosophila. Mol Biol Evol 16:1439-1456.

Ashburner M, Bodmer M, Lemeunier F (1984) On the evolutionary relationships of Drosophila melanogaster. Dev Genet 4:295-312.

Ashburner M, Golic KG, Hawley RS (2005) Drosophila: A Laboratory Handbook, 2nd edn. Cold Spring Harbor Laboratory Press, Cold Spring Harbor, NY.

Bächli G (1999-2005) The database on taxonomy of Drosophilidae. Available at: http://taxodros.unizh.ch/.

Baker RH, DeSalle R (1997) Multiple sources of character information and the phylogeny of Hawaiian drosophilids. Syst Biol 46:654-673.

Barker K, Lanyon SM (2000) The impact of parsimony weighting schemes on inferred relationships among toucans and neotropical barbets (Aves: Piciformes). Mol Phylogenet Evol 15:215-234.

Bock IR (1978) The bipectinata complex: a study in interspecific hybridization in the genus Drosophila (Insecta: Diptera). Aust J Biol Sci 31:197-208.

Bock IR (1980) Current status of the Drosophila melanogaster species group (Diptera). Syst Entomol 5:341-356.

Bock IR (1984) Interspecific hybridization in the genus Drosophila. Evol Biol 18:41-67.

Bock IR, Wheeler MR (1972) The Drosophila melanogaster species group. Univ Tex Publ 7213:1-102.

Bonacum J, O'Grady PM, Kambysellis M, DeSalle R (2005) Phylogeny and age of diversification of the planitibia species group of the Hawaiian Drosophila. Mol Phylogenet Evol 37:73-82.

Bremer K (1994) Branch support and tree stability. Cladistics 10:295-304.

Brown NM, Prager EM, Wang A, Wilson AC (1982) Mitochondrial DNA sequences of primates: tempo and mode of evolution. J Mol Evol 18:839-843.

Cariou M-L (1987) Biochemical phylogeny of the eight species in the Drosophila melanogaster subgroup, including $D$. sechellia and D. orena. Gen Res Camb 50:181-185.

Cariou M-L, Silvain J-F, Daubin V, Da Lage J-L, Lachaise D (2001) Divergence between Drosophila santomea and allopatric or sympatric populations of D. yakuba using paralogous amylase genes and migration scenarios along the volcanic line. Mol Ecol 10:649-660.

Coyne JA, Orr HA (2004) Speciation. Sinauer Associates, Sunderland.

Da Lage J-L, Wegnez M, Cariou M-L (1996) Distribution and evolution of introns in Drosophila amylase genes. J Mol Evol 43:334-347.

Da Lage J-L, Renard E, Chartois F, Lemeunier F, Cariou M-L (1998) Amyrel, a paralogous gene of the amylase gene family in Drosophila melanogaster and the Sophophora subgenus. Proc Natl Acad Sci USA 95:6848-6853.

Da Lage J-L, Van Wormhoudt A, Cariou M-L (2002) Diversity and evolution of the alpha-amylase genes in animals. Biologia (Bratisl) 57:181-189.

Delsuc F, Brinkmann H, Philippe H (2005) Phylogenomics and the reconstruction of the tree of life. Nature Rev Genet 6:361-375.

DeSalle R (1992) The phylogenetic relationships of flies in the family Drosophilidae deduced from mtDNA sequences. Mol Phylogenet Evol 1:31-40.

(C) 2007 The Authors J Zool Syst Evol Res (2007) 45(1), 47-63 Journal compilation (C) 2007 Blackwell Verlag, Berlin
DeSalle R, Brower AV (1997) Process partitions, congruence, and the independence of characters: inferring relationships among closely related Hawaiian drosophila from multiple gene regions. Syst Biol 46:751-764.

DeSalle R, Grimaldi DA (1993) Phylogenetic pattern and developmental process in drosophila. Syst Biol 42:458-475.

Durando CM, Baker RH, Etges WJ, Heed WB, Wesserman M, DeSalle R (2000) Phylogenetic analysis of the repleta species group of the genus Drosophila using multiple sources of characters. Mol Phylogenet Evol 16:296-307.

Galtier N, Gouy M (1995) Inferring phylogenies from DNA sequences of unequal base composition. Proc Natl Acad Sci USA 92:1131711321.

Galtier N, Gouy M (1998) Inferring pattern and process: maximumlikelihood implementation of a nonhomogeneous model of DNA sequence evolution for phylogenetic analysis. Mol Biol Evol 15:871879 .

Gleason JM, Powell JR (1997) Interspecific and intraspecific comparisons of the period locus in the Drosophila willistoni sibling species. Mol Biol Evol 14:741-753.

Gloor G, Engels W (1992) Single fly DNA preps for PCR. Drosoph Inf Serv 71:148-149.

Gompell N, Carroll SB (2003) Genetic mechanisms and constraints governing the evolution of correlated traits in drosophilid flies. Nature 424:931-935.

Gontcharov AA, Marin B, Melkonian M (2004) Are combined analyses better than single gene phylogenies? A case study using SSU rDNA and $r b c \mathrm{~L}$ sequence comparisons in the Zygnematophyceae (Streptophyta). Mol Biol Evol 21:612-624.

Goto SG, Kimura MT (2001) Phylogenetic utility of mitochondrial COI and nuclear Gpdh genes in Drosophila. Mol Phylogenet Evol 18:404-422.

Grimaldi DA (1990) A phylogenetic, revised classification of the genera in the Drosophilidae (Diptera). Bull Am Mus Nat Hist 197:1-139.

Gu X, Fu Y-X, Li W-H (1995) Maximum likelihood estimation of the heterogeneity of substitution rate among nucleotide sites. Mol Biol Evol 12:546-557.

Guindon S, Gascuel O (2003) A simple, fast, and accurate algorithm to estimate large phylogenies by maximum likelihood. Syst Biol 52:696-704.

Hillis DM, Huelsenbeck JP, Cunningham CW (1994) Application and accuracy of molecular phylogenies. Science 264:671-677.

Hillis DM, Pollock DD, McGuire JA, Zwickl DJ (2003) Is sparse taxon sampling a problem for phylogenetic inference? Syst Biol 52:124-126.

Hsu TC (1949) The external genital apparatus of male Drosophilidae in relation to systematics. Univ Tex Publ 4920:80-142.

Huelsenbeck JP, Ronquist F (2001) MrBayes: Bayesian inference of phylogeny. Bioinformatics 17:754-755.

Inomata N, Tachida H, Yamazaki T (1997) Molecular evolution of the Amy multigenes in the subgenus Sophophora of Drosophila. Mol Biol Evol 14:942-950.

Kastanis P, Eliopoulos E, Goulielmos GN, Tsakas S, Loukas M (2003) Macroevolutionary relationships of species of Drosophila melanogaster group based on mtDNA sequences. Mol Phylogenet Evol 28:518-528.

Katoh T, Tamura K, Aotsuka T (2000) Phylogenetic position of the subgenus Lordiphosa of the genus Drosophila (Diptera: Drosophilidae) inferred from alcohol dehydrogenase $(A d h)$ gene sequences. J Mol Evol 51:122-130.

Kliman RM, Andolfatto P, Coyne JA, Depaulis F, Kreitman M, Berry AJ, McCarter J, Wakeley J, Hey J (2000) The population genetics of the origin and divergence of the Drosophila simulans complex species. Genetics 156:1913-1931.

Ko W, David R, Akashi H (2003) Molecular phylogeny of the Drosophila melanogaster subgroup. J Mol Evol 57:562-573.

Kopp A (2006) Basal relationships in the Drosophila melanogaster species group. Mol Phylogenet Evol 39:787-798.

Kopp A, True JR (2002) Phylogeny of the oriental Drosophila melanogaster species group: a multilocus reconstruction. Syst Biol 51:786-805. 
Kwiatowski J, Ayala FJ (1999) Phylogeny of drosophila and related genera: conflict between molecular and anatomical analyses. Mol Phylogenet Evol 13:319-328.

Lachaise D, Silvain J-F (2004) How two Afrotropical endemics made two cosmopolitan human commensals from Africa: the Drosophila melanogaster $-D$. simulans riddle. Genetica 120:17-39.

Lachaise D, Cariou M-L, David JR, Lemeunier F, Tsacas L, Ashburner M (1988) Historical biogeography of the Drosophila melanogaster species subgroup. Evol Biol 22:159-225.

Lachaise D, Harry M, Solignac M, Lemeunier F, Bénassi V, Cariou M-L (2000) Evolutionary novelties in islands: Drosophila santomea, a new melanogaster sister species from São Tomé. Proc Roy Soc London 267:1487-1495.

Lachaise D, Capy P, Cariou M-L, Joly D, Lemeunier F, David JR (2004) Nine relatives from one African ancestor: the population biology of the Drosophila melanogaster subgroup species. In: Singh RS, Uyenoyama MK (eds), The Evolution of Population Biology. Cambridge University Press, Cambridge, pp. 315-343.

Lanave C, Preparata GCS, Serio G (1984) A new method for calculating evolutionary substitution rates. J Mol Evol 20:86-93.

Lemeunier F, Tsacas L, David J, Ashburner M (1986) The melanogaster species group. In: Thomson JR, Carson HL (eds), The Genetics and Biology of Drosophila, Vol. 3e. Academic Press, New York, pp. 147-256.

Lemeunier F, Aulard S, Arienti M, Jallon J-M, Cariou M-L, Tsacas L (1997) The ercepeae complex: new cases of insular speciation within the Drosophila ananassae species subgroup (melanogaster group) and descriptions of two new species (Diptera: Drosophilidae). Ann Entomol Soc Am 90:28-42.

Lewis RL, Beckenbach AT, Mooers AO (2005) The phylogeny of the subgroups within the melanogaster species group: likelihood tests on $\mathrm{COI}$ and COII sequences and a Bayesian estimate of phylogeny. Mol Phylogenet Evol 37:15-24.

Maczkowiak F, Da Lage J-L (2006) Origin and evolution of the Amyrel gene in the $\boldsymbol{\alpha}$-amylase family of Diptera. Genetica 128: 145-158.

May-Collado L, Agnarsson I (2006) Cytochrome b and Bayesian inference of whale phylogeny. Mol Phylogenet Evol 38: 344-354.

O'Grady PM, Kidwell MG (2002) Phylogeny of the subgenus Sophophora (Diptera: Drosophilidae) based on combined analysis of nuclear and mitochondrial sequences. Mol Phylogenet Evol 22:442-453.

O'Grady PM, Clark JB, Kidwell MG (1998) Phylogeny of the Drosophila saltans species group based on combined analysis of nuclear and mitochondrial DNA sequences. Mol Biol Evol 15:656-664.

Pélandakis M, Solignac M (1993) Molecular phylogeny of Drosophila based on ribosomal RNA sequences. J Mol Evol 37:525-543.

Pélandakis M, Higgins DG, Solignac M (1991) Molecular phylogeny of the subgenus Sophophora of Drosophila derived from large subunit of ribosomal RNA sequences. Genetica 84:87-94.

Posada D, Crandall KA (1998) MODELTEST: testing the model of DNA substitution. Bioinformatics 14:817-818.

Powell JR (1997) Progress and Prospects in Evolutionary Biology. The Drosophila Model. Oxford University Press, New York.

Prigent S, Renard E, Cariou M-L (2003) Electrophoretic mobility of amylase in Drosophilids indicates adaptation to ecological diversity. Genetica 119:133-145.

Rafael V (1984) Relations interspécifiques dans le nouveau complexe africain de Drosophila bakoue du groupe melanogaster, sous-groupe montium (Diptera, Drosophilidae). Bull Soc Zool Fr 109:179-189.

Remsen J, DeSalle R (1998) Character congruence of multiple data partitions and the origin of the Hawaiian Drosophilidae. Mol Phylogenet Evol 9:225-235.

Remsen J, O'Grady P (2002) Phylogeny of Drosophilinae (Diptera: Drosophilidae), with comments on combined analysis and character support. Mol Phylogenet Evol 24:249-264.

Robe LJ, Valente VLS, Budnik M, Loreto ELS (2005) Molecular phylogeny of the subgenus Drosophila (Diptera, Drosophilidae) with an emphasis on Neotropical species and groups: a nuclear versus mitochondrial approach. Mol Phylogenet Evol 36:623-640.
Rodriguez-Trelles F, Tarrio R, Ayala FJ (2000) Evidence for a high ancestral GC content in Drosophila. Mol Biol Evol 17:1710-1717.

Russo CAM, Takezaki N, Nei M (1995) Molecular phylogeny and divergence times of Drosophilid species. Mol Biol Evol 12:391-404.

Schawaroch V (2002) Phylogeny of a paradigm lineage: the Drosophila melanogaster species group (Diptera: Drosophilidae). Biol J Linn Soc 76:21-37.

Sorenson MD (1999) TreeRot, version 2. Boston University, Boston. Spicer GS, Bell CD (2002) Molecular phylogeny of the Drosophila virilis species group (Diptera: Drosophilidae) inferred from mitochondrial $12 \mathrm{~S}$ and 16S ribosomal RNA genes. Ann Entomol Soc Am 95:156-161.

Swofford DL (2002) PAUP*. Phylogenetic Analysis Using Parsimony* and Other Methods. Version 4.0b10. Sinauer Associates, Sunderland, MA.

Tamura K, Subramanian S, Kumar S (2004) Temporal patterns of fruit fly (Drosophila) evolution revealed by mutation clocks. Mol Biol Evol 21:36-44.

Tarrio R, Rodriguez-Trelles F, Ayala FJ (2001) Shared nucleotide composition biases among species and their impact on phylogenetic reconstructions of the Drosophilidae. Mol Biol Evol 18:1464-1473.

Tatarenkov A, Ayala FJ (2001) Phylogenetic relationships among species groups of the virilis-repleta radiation of Drosophila. Mol Phylogenet Evol 21:327-331.

Tatarenkov A, Kwiatowski J, Skarecky D, Barrio E, Ayala FJ (1999) On the evolution of Dopa decarboxylase $(D d c)$ and Drosophila systematics. J Mol Evol 48:445-462.

Thompson JD, Higgins DG, Gibson TJ (1994) CLUSTAL W: improving the sensitivity of progressive multiple sequence alignment through sequence weighting, positions-specific gap penalties and weight matrix choice. Nucleic Acids Res 22:4673-4680.

Throckmorton LH (1975) The phylogeny, ecology and geography of Drosophila. In: King RC (ed), Handbook of Genetics. Plenum Press, New York, pp. 421-469.

Tobari YN (1993) Drosophila ananassae, Genetical and Biological Aspects. Japan Scientific Societies Press, Tokyo, 289 p.

Toda MJ (1991) Drosophilidae (Diptera) in Myanmar (Burma) VII. The Drosophila melanogaster species-group, excepting the D. montium species-subgroup. Orient Insects 25:69-94.

Tsacas L, Chassagnard M-T (1992) L'identité de Drosophila (Sophophora) vulcana Graber (Diptera, Drosophilidae). Bull Soc Entomol Fr 96:427-432.

Tsacas L, David J (1977) Systematics and biogeography of the Drosophila kikkawai complex, with description of new species (Diptera, Drosophilidae). Ann Soc Entomol Fr (N.S.) 13:675-693.

Wu C-I (2001) The genic view of the process of speciation. J Evol Biol 14:851-865.

Yang Z (1994) Estimating the pattern of nucleotide substitution. J Mol Evol 39:105-111.

Yang Y, Zhang Y-P, Qian Y-H, Zeng Q-T (2004) Phylogenetic relationships of Drosophila melanogaster species group deduced from spacer regions of histone gene H2A-H2B. Mol Phylogenet Evol 30:336-343.

Zhang Z, Inomata N, Cariou M-L, Da Lage J-L, Yamazaki T (2003) Phylogeny and evolution of the amylase multigenes in Drosophila montium species subgroup. J Mol Evol 56:121-130.

Zwickl DJ, Hillis DM (2002) Increased taxon sampling greatly reduces phylogenetic error. Syst Biol 51:588-598.

Authors'addresses: Jean-Luc Da Lage (for correspondence), MarieLouise Cariou and Daniel Lachaise (deceased), UPR 9034 Evolution, génomes et spéciation, CNRS, 91198 Gif sur Yvette cedex, France. E-mail: jldl@legs.cnrs-gif.fr, marie-louise.cariou@legs.cnrs-gif.fr; Gaël Joseph Kergoat, INRA, Centre de Biologie et de Gestion des Populations, Campus International de Baillarguet, CS 30016, 34988 Montferrier-sur-Lez, France. E-mail: kergoat@ensam.inra.fr; Frédérique Maczkowiak, Institut Curie, 91400 Orsay, France. E-mail: maczkowiak@curie.u-psud.fr; Jean-François Silvain, IRD, UR R072, c/o CNRS UPR 9034, 91198 Gif sur Yvette cedex, France. E-mail: jean-francois.silvain@legs.cnrs-gif.fr 